\title{
FIXED POINT INDICES AND FIXED WORDS AT INFINITY OF SELFMAPS OF GRAPHS
}

\author{
QIANG ZHANG, XUEZHI ZHAO
}

\begin{abstract}
Indices of fixed point classes play a central role in Nielsen fixed point theory. Jiang-Wang-Zhang proved that for selfmaps of graphs and surfaces, the index of any fixed point class has an upper bound called its characteristic.

In this paper, we study the difference between the index and the characteristic for selfmaps of graphs. First, for free groups, we extend attracting fixed words at infinity of automorphisms into that of injective endomorphisms. Then, by using relative train track technique, we show that the difference mentioned above is quite likely to be the number of equivalence classes of attracting fixed words of the endomorphism induced on the fundamental group. Since both of attracting fixed words and the existed characteristic are totally determined by endomorphisms themselves, we give a new algebraic approach to estimate indices of fixed point classes of graph selfmaps.

As consequence, we obtain an upper bound for attracting fixed words of injective endomorphisms of free groups, generalizing the one for automorphisms due to Gaboriau-JaegerLevitt-Lustig. Furthermore, we give a simple approach to roughly detecting whether fixed words exist or not.
\end{abstract}

\section{INTRODUCTION}

Fixed point theory studies the fixed points of a selfmap $f$ of a space $X$. Nielsen fixed point theory, in particular, is concerned with the properties of the fixed point set

$$
\text { Fix } f:=\{x \in X \mid f(x)=x\},
$$

that are invariant under homotopy of the selfmap $f$ (see [J1] for an introduction to Nielsen fixed point theory).

The fixed point set Fix $f$ splits into a disjoint union of fixed point classes: two fixed points $x$ and $x^{\prime}$ are said to be in the same class if and only if they can be joined by a Nielsen path which is a path homotopic (relative to endpoints) to its own $f$-image. Let $\operatorname{Fpc}(f)$ denote the set of all the fixed point classes of $f$. For each fixed point class $\mathbf{F} \in \operatorname{Fpc}(f)$, a homotopy invariant index $\operatorname{ind}(f, \mathbf{F}) \in \mathbb{Z}$ is well-defined. A fixed point class is essential if its index is non-zero. For alternative definitions of fixed point class by covering spaces, see Section 3.1

Date: May 26, 2020.

2010 Mathematics Subject Classification. 55M20, 55N10, 32Q45.

Key words and phrases. Index, attracting fixed point, fixed subgroups, graph selfmap, free group.

The authors are partially supported by NSFC (Nos. 11771345, 11961131004 and 11971389) and Capacity Building for Sci-Tech Innovation-Fundamental Scientific Research Funds. 
In the paper [JWZ], B. Jiang, S. Wang and Q. Zhang defined a new homotopy invariant rank $\operatorname{rk}(f, \mathbf{F}) \in \mathbb{N}$ for a fixed point class $\mathbf{F}$ of $f$. For an endomorphism $\phi: G \rightarrow G$ of a group $G$, its fixed subgroup refers to the subgroup

$$
\operatorname{Fix}(\phi):=\{g \in G \mid \phi(g)=g\} \leq G .
$$

The stabilizer of a fixed point $x \in \mathbf{F}$ is the subgroup $\operatorname{Stab}(f, x):=\operatorname{Fix}\left(f_{\pi}\right) \subset \pi_{1}(X, x)$, where $f_{\pi}: \pi_{1}(X, x) \rightarrow \pi_{1}(X, x)$ is the induced endomorphism. Since it is independent of the choice of $x \in \mathbf{F}$, up to isomorphism, the stabilizer of a fixed point class $\mathbf{F}$ is defined as $\operatorname{Stab}(f, \mathbf{F}):=\operatorname{Stab}(f, x)$, for any $x \in \mathbf{F}$. The rank of $\mathbf{F}$ is defined as

$$
\operatorname{rk}(f, \mathbf{F}):=\operatorname{rkStab}(f, \mathbf{F}),
$$

where the rank of a group is the minimal number of generators.

For a selfmap $f$ of a surface or a graph $X$, they defined the characteristic of a fixed point class $\mathbf{F}$ of $f$ as

$$
\operatorname{chr}(f, \mathbf{F}):=1-\operatorname{rk}(f, \mathbf{F}),
$$

with the only exception that $\operatorname{chr}(f, \mathbf{F}):=\chi(X)=2-\operatorname{rk}(f, \mathbf{F})$ when $X$ is a closed surface and $\operatorname{Stab}(f, \mathbf{F})=\pi_{1}(X)$. What is more, some relations between the characteristic and the index of fixed point classes of graphs and surfaces are proved.

Theorem 1.1 (Jiang-Wang-Zhang, [JWZ]). Suppose $X$ is either a connected finite graph or a connected compact hyperbolic surface, and $f: X \rightarrow X$ is a selfmap. Then

(A) $\operatorname{ind}(f, \mathbf{F}) \leq \operatorname{chr}(f, \mathbf{F})$ for every fixed point class $\mathbf{F}$ of $f$;

(B) when $X$ is not a tree,

$$
\sum_{\operatorname{ind}(f, \mathbf{F})+\operatorname{chr}(f, \mathbf{F})<0}\{\operatorname{ind}(f, \mathbf{F})+\operatorname{chr}(f, \mathbf{F})\} \geq 2 \chi(X),
$$

where the sum is taken over all fixed point classes $\mathbf{F}$ with $\operatorname{ind}(f, \mathbf{F})+\operatorname{chr}(f, \mathbf{F})<0$.

An analogue on 3-manifolds can be found in [Z].

In this paper, we are primarily interested in a $\pi_{1}$-injective selfmap $f: X \rightarrow X$ of a graph $X$, i.e., $f$ induces an injective endomorphism $f_{\pi}$ of the fundamental group $\pi_{1}(X)$. In this setting, we will introduce a new homotopy invariant $a(f, \mathbf{F}) \in \mathbb{N}$ (see Definition 3.1) for each fixed point class $\mathbf{F}$ of $f$. Thanks to this new invariant, we will improve the inequality (A) of Theorem 1.1 into an inequality (equality when $\chi(X) \geq-1$ ) among $\operatorname{ind}(f, \mathbf{F}), \operatorname{rk}(f, \mathbf{F})$ and $a(f, \mathbf{F})$. For convenience, we define the improved characteristic of a fixed point class $\mathbf{F}$ of $f$ as $\operatorname{ichr}(f, \mathbf{F}):=\operatorname{chr}(f, \mathbf{F})-a(f, \mathbf{F})$, namely,

$$
\operatorname{ichr}(f, \mathbf{F}):=1-\operatorname{rk}(f, \mathbf{F})-a(f, \mathbf{F}) .
$$

Note that $\operatorname{ichr}(f, \mathbf{F}) \leq 1$ and only depends on the induced endomorphism $f_{\pi}$ of $\pi_{1}(X)$.

For brevity, we will write ind $(\mathbf{F}), \operatorname{rk}(\mathbf{F}), a(\mathbf{F}), \operatorname{chr}(\mathbf{F})$ and $\operatorname{ichr}(\mathbf{F})$ if no confusion exists for the selfmap $f$ in the context. In Nielsen fixed point theory, a fixed point class $\mathbf{F}$ is allowed to be empty. In that case the above definitions of $\operatorname{rk}(\mathbf{F}), a(\mathbf{F})$ and $\operatorname{ichr}(\mathbf{F})$ do 
not make sense. Alternative approaches using covering spaces and paths, will be given in Section 3

The main results of this paper are the following.

Theorem 1.2. Let $X$ be a connected finite graph and $f: X \rightarrow X$ be a $\pi_{1}$-injective selfmap. Then for every fixed point class $\mathbf{F}$ of $f$, we have

$$
\operatorname{ind}(\mathbf{F}) \leq \operatorname{ichr}(\mathbf{F}) .
$$

When the Euler characteristic $\chi(X) \geq 0$, the equality ind $(\mathbf{F})=\operatorname{ichr}(\mathbf{F})$ holds immediately, see Example 3.8 When $\chi(X)=-1$, we have

Theorem 1.3. If $X$ is a connected finite graph with Euler characteristic $\chi(X)=-1$ and $f: X \rightarrow X$ is a $\pi_{1}$-injective selfmap, then for every essential fixed point class $\mathbf{F}$ of $f$, we have

$$
\operatorname{ind}(\mathbf{F})=\operatorname{ichr}(\mathbf{F}),
$$

and for every inessential fixed point class $\mathbf{F}$, we have $0=\operatorname{ind}(\mathbf{F}) \leq \operatorname{ichr}(\mathbf{F}) \leq 1$.

Conjecture 1.4. The equality ind $(\mathbf{F})=\operatorname{ichr}(\mathbf{F})$ always holds in Theorem 1.2

In other words, we conjecture that ind $(\mathbf{F})=1-\operatorname{rk}(\mathbf{F})-a(\mathbf{F})$, for some evidence, see Section 6 This equality will give a new algebraic approach for computing indices of fixed point classes of $\pi_{1}$-injective selfmaps of graphs.

Remark 1.5. For any selfmap $f: X \rightarrow X$ of a connected finite graph $X$, if $f$ is not $\pi_{1}$-injective, by [J2] Lemma A], it is a mutant (see [J2] for a definition) of a $\pi_{1}$-injective selfmap $g$ of a connected finite graph $Y$ with $\chi(Y) \geq \chi(X)$. Since mutants have the same set of indices of essential fixed point classes, we can also compute the index by $\operatorname{ichr}(\mathbf{F})$ for an essential fixed point class $\mathbf{F}$ of $f$ if Conjecture 1.4 is true. Indeed, let $\mathbf{F}^{\prime} \in \operatorname{Fpc}(g)$ be the corresponding fixed point class of $\mathbf{F}$, then

$$
\operatorname{ind}(f, \mathbf{F}) \stackrel{\text { mutant invar. }}{=} \operatorname{ind}\left(g, \mathbf{F}^{\prime}\right) \stackrel{\text { Conj. 1.4. }}{=} \operatorname{ichr}\left(g, \mathbf{F}^{\prime}\right) .
$$

Replacing ind $(\mathbf{F})$ with ichr $(\mathbf{F})$ in the inequality (B) of Theorem 1.1 as a corollary of Theorem 1.2 we have $2 \chi(X)-1 \leq \operatorname{ichr}(\mathbf{F}) \leq 1$ and the following bound immediately.

Corollary 1.6. Suppose $X$ is a connected finite graph but not a tree, and $f: X \rightarrow X$ is a $\pi_{1}$-injective selfmap. Then

$$
\sum_{\mathbf{F} \in \operatorname{Fpc}(f)} \max \{0, \operatorname{rk}(\mathbf{F})+a(\mathbf{F}) / 2-1\} \leq-\chi(X),
$$

where the sum is taken over all fixed point classes $\mathbf{F}$ of $f$.

For an injective endomorphism $\phi: F_{n} \rightarrow F_{n}$ of a free group $F_{n}$ of rank $n \geq 1$, it induces an endomorphism $\phi^{\mathrm{ab}}$ of the abelianization of $F_{n}$,

$$
\phi^{\mathrm{ab}}: \mathbb{Z}^{n} \rightarrow \mathbb{Z}^{n}
$$


Let $\operatorname{tr}\left(\phi^{\mathrm{ab}}\right)$ be the trace of a matrix of $\phi^{\mathrm{ab}}$. For any $c \in F_{n}$, let $i_{c}: F_{n} \rightarrow F_{n}, g \mapsto c g c^{-1}$ be the inner automorphism induced by $c$. Let $a(\phi)$ be the number of equivalence classes of attracting fixed points for the action of $\phi$ on the boundary of $F_{n}$, see Definition 2.4

Using the trace $\operatorname{tr}\left(\phi^{\mathrm{ab}}\right)$, we have the following theorem giving an approach to roughly detecting whether fixed words exist or not.

Theorem 1.7. Let $\phi$ be an injective endomorphism of a free group $F_{n}$. Then there exists $c \in F_{n}$ such that

$$
\operatorname{rkFix}\left(i_{c} \circ \phi\right)=a\left(i_{c} \circ \phi\right)=0
$$

if the trace $\operatorname{tr}\left(\phi^{\mathrm{ab}}\right)<1$; and

$$
\operatorname{rkFix}\left(i_{c} \circ \phi\right)+a\left(i_{c} \circ \phi\right)>1
$$

if $n \leq 2$ and $\operatorname{tr}\left(\phi^{\mathrm{ab}}\right)>1$.

Moreover, we obtain an upper bound for the rank of the fixed subgroup and the number $a(\phi)$ of an injective endomorphism $\phi$ of a free group.

Theorem 1.8. Let $\phi$ be any injective endomorphism of a free group $F_{n}$. Then

$$
\operatorname{rkFix}(\phi)+a(\phi) / 2 \leq n
$$

In [GJLL], Gaboriau, Jaeger, Levitt and Lustig proved the inequality above for automorphisms of $F_{n}$, by using groups acting on $\mathbb{R}$-trees. Our proof for general case is based on Theorem 1.2 and Bestvina-Handel's train track maps.

The paper is organized as follows. In Section 2 we introduce attracting fixed points and $a(\phi)$ for any injective endomorphism $\phi$ of a free group. In Section 3 we first give the background of Nielsen theory and define the improved characteristic for fixed point classes of selfmaps of graphs, and then show some invariance of that. In Section 4 we study the improved characteristic of selfmaps of graphs by an approach using train track maps. In Section 5, we complete the proofs of Theorem 1.2, Theorem 1.3, Theorem 1.7 and Theorem 1.8 Finally in Section 6 , we give some examples supporting Conjecture 1.4

Acknowledgements. The authors would like to thank Alexander Fel'shtyn for helpful discussions, and thank Shida Wang for detailed comments.

\section{ATtRACTING FIXED WORDS AND $a(\phi)$ OF FREE GROUPS}

In [GJLL, Sect. 1], the authors introduced attracting fixed words and the number $a(\phi)$ of an automorphism $\phi$ of a free group. In this section, we will extend that to injective endomorphisms of free groups. 
2.1. Attracting fixed word and attracting fixed point. Let $F$ be a free group of rank $n$. Fixed a basis (i.e., a free generating set) $\Lambda=\left\{g_{1}, \ldots, g_{n}\right\}$ for $F$, we view $F$ as the set of reduced words in the letters $g_{i}^{ \pm 1}$, and $\partial F$ as the set of infinite reduced words $W=w_{1} w_{2} \cdots w_{i} \cdots$, i.e., $w_{i} \in \Lambda^{ \pm}=\left\{g_{1}^{ \pm 1}, \ldots, g_{n}^{ \pm 1}\right\}$ and $w_{i} \neq w_{i+1}^{-1}$. Denote $W_{i}=$ $w_{1} \cdots w_{i}$. The word length of a word $W \in F$ with respect to $\Lambda$ is written $|W|$. Given two finite or infinite reduced words $W, V \in \bar{F}:=F \sqcup \partial F$, let $W \wedge V$ be the longest common initial segment of $W$ and $V$. The initial segment metric $d_{i . s}$ on $\bar{F}$ is defined by $d_{i . s}(W, W)=0$ and for $W \neq V$,

$$
d_{i . s}(W, V)=\frac{1}{1+|W \wedge V|} .
$$

With this metric, $\bar{F}$ is compact (called end completion as in $[\bar{C}]$ or compactification as a hyperbolic group in the sense of Gromov), and $F$ is dense in $\bar{F}$. The boundary $\partial F$ is a compact space that is homeomorphic to a Cantor set when $n \geq 2$. A sequence of reduced words $V_{p} \in \bar{F}$ converges to an infinite word $W \in \partial F$ if and only if $\lim _{p \rightarrow+\infty}\left|W \wedge V_{p}\right|=$ $+\infty$.

The natural actions of $F$ and $\operatorname{Aut}(F)$ on $F$ extend continuously to $\bar{F}$ : a left multiply $W: F \rightarrow F$ by a word $W \in F$ and an automorphism $f: F \rightarrow F$ extend uniquely to homeomorphisms $W: \partial F \rightarrow \partial F$ and $\bar{f}: \partial F \rightarrow \partial F$ (see $[\overline{\mathrm{C}}]$ ), respectively. Any finitely generated subgroup $F^{\prime}<F$ is quasi-convex [Sh1], and hence an inclusion induces a natural embedding $\partial F^{\prime} \hookrightarrow \partial F$ ([CDP p. 115]). For an injective endomorphism $\phi$ : $F \rightarrow F$, since $F \cong \phi(F)<F$, we have $\partial F \cong \partial(\phi(F)) \hookrightarrow \partial F$. Therefore

Lemma 2.1. Let $\phi: F \rightarrow F$ be an injective endomorphism of $F$. Then $\phi$ can be extended to a continuous injective map $\bar{\phi}: \partial F \rightarrow \partial F$.

For now on, let $\phi: F \rightarrow F$ be an injective endomorphism of $F$. By [DV] p.32, Lem II.2.4], there is a cancelation bound $\mathcal{B}>0$ for $\phi$ :

$$
|\phi(W \cdot V)| \geq|\phi(W)|+|\phi(V)|-2 \mathcal{B},
$$

whenever $W, V \in F$ are finite reduced words, and $W \cdot V$ denote the product $W V$ if there is no cancelation between $W$ and $V$, i.e., $|W \cdot V|=|W|+|V|$.

Let $W=w_{1} \cdots w_{i} \cdots \in \partial F$ be a fixed infinite reduced word of $\phi$. Write

$$
\phi\left(W_{i}\right)=W_{k(i)} \cdot V_{i}
$$

with $W_{k(i)}=W \wedge \phi\left(W_{i}\right)$ and hence $k(i)=\left|W \wedge \phi\left(W_{i}\right)\right|$. Since $W$ is fixed by $\phi$, the sequence $k(i) \rightarrow+\infty$ as $i$ increases. Bounded cancelation implies $\left|V_{i}\right| \leq \mathcal{B}$, and $|k(i+1)-k(i)|$ is bounded by the constant $\max \left\{\phi\left(g_{1}\right), \ldots, \phi\left(g_{n}\right)\right\}$ depending only on $\phi$. A fixed infinite word $W$ is said to be an attracting fixed word of $\phi$ if

$$
\lim _{i \rightarrow+\infty}\left|W \wedge \phi\left(W_{i}\right)\right|-i=+\infty
$$

Note that there exists $i_{0}>0$ such that for all $i \geq i_{0}$, we have $k(i) \geq i+\mathcal{B}+1$. For any reduced word $W^{\prime} \in \bar{F}$, let $W_{i}=W \wedge W^{\prime}$ and $W^{\prime}=W_{i} \cdot V^{\prime}$, then $\phi\left(W^{\prime}\right)=\phi\left(W_{i}\right) \phi\left(V^{\prime}\right)$ 
and the cancelation length between $\phi\left(W_{i}\right)=W_{k(i)} \cdot V_{i}$ and $\phi\left(V^{\prime}\right)$ is bounded by $\mathcal{B}$. Therefore, if $\left|W \wedge W^{\prime}\right|=i \geq i_{0}$, then $\left|W \wedge \phi\left(W^{\prime}\right)\right| \geq k(i)-\mathcal{B}>i=\left|W \wedge W^{\prime}\right|$. So

Lemma 2.2. Let $W$ be an attracting fixed word of $\phi$. Then there exists an integer $i_{0}$ such that $\left|W \wedge \phi\left(W^{\prime}\right)\right|>\left|W \wedge W^{\prime}\right|$ for any word $W^{\prime}$ with $\left|W \wedge W^{\prime}\right| \geq i_{0}$.

We say that a fixed infinite word $W$ is an attracting fixed point of $\phi$ if there exists a neighborhood $\mathcal{U}$ of $W \in \bar{F}$ such that

$$
W^{\prime} \in \mathcal{U} \Longrightarrow \lim _{p \rightarrow+\infty} \phi^{p}\left(W^{\prime}\right)=W .
$$

Note that by the definition of the initial segment metric on $\bar{F}, W^{\prime} \in \mathcal{U}$ if and only if $\left|W \wedge W^{\prime}\right|>r^{-1}-1$, where $r<1$ is the radium of $\mathcal{U}$.

By a similar discuss as in the proof of [GJLL, Proposition 1.1], we have

Proposition 2.3. Let $W \in \partial F$ be a fixed infinite word of $\phi$. If $W$ is an attracting fixed word of $\phi$, then $W \notin \partial(\operatorname{Fix} \phi)$. Moreover, $W$ is an attracting fixed point of $\phi$ if and only if it is an attracting fixed word of $\phi$.

Proof. Let $W$ be an attracting fixed word of $\phi$. If $W \in \partial(\operatorname{Fix} \phi)$, we can write $W=V_{1}$. $V_{2} \cdots$ and $W_{s_{i}}=V_{1} \cdots V_{i}$, where the reduced finite words $V_{i} \in \operatorname{Fix} \phi$. Then $\phi\left(W_{s_{i}}\right)=$ $W_{s_{i}}$ and $k\left(s_{i}\right)=s_{i}$ for all $i$, contradicting the hypothesis that $W$ is attracting. Therefore, $W \notin \partial(\operatorname{Fix} \phi)$. Moreover, pick $i_{0}$ as in Lemma 2.2 and $\mathcal{U}$ a neighborhood with radium $<i_{0}^{-1}$. For any $W^{\prime} \in \mathcal{U}, d_{i . s}\left(W, W^{\prime}\right)=\left(1+\left|W \wedge W^{\prime}\right|\right)^{-1}<i_{0}^{-1}$, hence $\left|W \wedge W^{\prime}\right| \geq i_{0}$. By Lemma2.2, we have $\left|W \wedge \phi\left(W^{\prime}\right)\right|>\left|W \wedge W^{\prime}\right|$, and hence $\lim _{p \rightarrow+\infty}\left|W \wedge \phi^{p}\left(W^{\prime}\right)\right|=$ $+\infty$, namely, $\lim _{p \rightarrow+\infty} \phi^{p}\left(W^{\prime}\right)=W$. Therefore, $W$ is an attracting fixed point of $\phi$.

Now let $W$ be an attracting fixed point of $\phi$. Then for $i$ large enough, we have $W=$ $\lim _{p \rightarrow+\infty} \phi^{p}\left(W_{i}\right)$. Consider the words $U_{i}=W_{i}^{-1} \phi\left(W_{i}\right)$. Note that

$$
U_{p}=U_{i} \quad \Longrightarrow \quad W_{p} W_{i}^{-1} \in \operatorname{Fix} \phi .
$$

If the sequence of words $U_{p}$ takes the same value infinity times, fix $i$ we get

$$
W=\lim _{p \rightarrow+\infty} W_{p}=\lim _{p \rightarrow+\infty} W_{p} W_{i}^{-1} \in \partial(\operatorname{Fix} \phi),
$$

contradicting the hypothesis that $W$ is an attracting fixed point. Therefore, $\lim _{p \rightarrow+\infty}\left|U_{p}\right|=$ $+\infty$. Recall that $\phi\left(W_{p}\right)=W_{k(p)} \cdot V_{p}$ with $\left|V_{p}\right| \leq \mathcal{B}$, we have

$$
|k(p)-p|+\mathcal{B} \geq\left|W_{p}^{-1} W_{k(p)} \cdot V_{p}\right|=\left|W_{p}^{-1} \phi\left(W_{p}\right)\right|=\left|U_{p}\right| \rightarrow+\infty
$$

as $p$ goes to infinity. Since $|k(p+1)-k(p)|$ is bounded, and $W$ is an attracting fixed point of $\phi$, we have $\lim _{p \rightarrow+\infty}(k(p)-p)=+\infty$, i.e., $W$ is an attracting fixed word of $\phi$.

2.2. The number $a(\phi)$ and similarity invariance. Now let us give the definition of the number $a(\phi)$ of equivalence classes of attracting fixed points.

Definition 2.4. Let $\phi: F \rightarrow F$ be an injective endomorphism of a free group $F$. We say that two fixed infinite words $W, W^{\prime} \in \partial F$ are equivalent if there exists a fixed word $U \in \operatorname{Fix}(\phi)$ such that $W^{\prime}=U W$. Note that any word equivalent to an attracting fixed 
word of $\phi$ is also an attracting fixed word of $\phi$. Let $\mathscr{A}(\phi)$ be the set of equivalence classes of attracting fixed words of $\phi$, and let $a(\phi)$ be the cardinality of $\mathscr{A}(\phi)$.

Remark 2.5. Let $\mathcal{A}(\phi)$ be the set of attracting fixed words of $\phi$. Then $\mathscr{A}(\phi)$ equals Fix $(\phi) \backslash \mathcal{A}(\phi)$, the set of orbits of Fix $(\phi)$ acting on $\mathcal{A}(\phi)$.

For a free group $F$, let $\operatorname{End}(F)($ resp. $\operatorname{Inn}(F), \operatorname{Inj}(F))$ be the set of endomorphisms (resp. inner automorphisms, injective endomorphisms) of $F$.

Consider the natural left action of $\operatorname{Inn}(F)$ on $\operatorname{End}(F)$. For an endomorphism $\phi \in$ $\operatorname{End}(F)$, we say that the orbit of $\phi$ is an Inn-coset, written $\operatorname{Inn} \phi$. Namely, two endomorphisms $\phi_{1}, \phi_{2} \in \operatorname{End}(F)$ are in the same Inn-coset if and only if there exists $m \in F$ such that $\phi_{2}=i_{m} \circ \phi_{1}$ with $i_{m}(g)=m g m^{-1}$ for any $g \in F$. We say that $\phi_{1}$ and $\phi_{2}$ are similar if $m$ can be written as $m=c \phi_{1}\left(c^{-1}\right)$ for some $c \in F$, or equivalently, if $\phi_{2}=i_{c} \circ \phi_{1} \circ\left(i_{c}\right)^{-1}$.

Note that every Inn-coset is a disjoint union of similarity classes, and the rank rkFix $(\phi)$ and the number $a(\phi)$ are both similarity invariants.

As an algebraic version of Corollary 1.6 we will prove the following stronger version of Theorem 1.8 in Section 5 .

Theorem 2.6. Let $\phi$ be an injective endomorphism of a free group $F_{n}$ of rank $n \geq 2$. Then

$$
\sum_{[\psi] \subset \operatorname{Inn} \phi} \max \{0, \operatorname{rkFix}(\psi)+a(\psi) / 2-1\} \leq n-1,
$$

where the sum runs over all distinct similarity classes $[\psi]$ contained in the Inn-coset $\operatorname{Inn} \phi$.

Example 2.7. Let $F$ be the free group of rank 1, i.e., $F=\langle g \mid-\rangle \cong \mathbb{Z}$. Then any endomorphism $\phi: F \rightarrow F$ has the form $\phi(g)=g^{k}$ with $\operatorname{tr}\left(\phi^{\mathrm{ab}}\right)=k$ for some integer $k$.

Note that the boundary $\partial F$ consists of two points: $g g \cdots g \cdots$ and $g^{-1} g^{-1} \cdots g^{-1} \cdots$. We have that

\begin{tabular}{|l|c|c|c|c|}
\hline $\operatorname{tr}\left(\phi^{\mathrm{ab}}\right)$ & $\phi(g)$ & $\operatorname{Fix}(\phi)$ & $\operatorname{rkFix}(\phi)$ & $a(\phi)$ \\
\hline 0 & 1 & $\{1\}$ & 0 & N/A \\
1 & $g$ & $\mathbb{Z}$ & 1 & 0 \\
$k>1$ & $g^{k}$ & $\{1\}$ & 0 & 2 \\
$k<0$ & $g^{k}$ & $\{1\}$ & 0 & 0 \\
\hline
\end{tabular}

The trivial endomorphism is not injective, and therefore $a(\phi)$ is not defined. For the identity endomorphism, each element in $F$ is fixed. It is obvious that the two infinite words are both fixed, but are not attracting.

\section{IMPROVED CHARACTERISTIC OF FIXED POINT CLASSES}

In [JWZ, Section 2], the authors gave some definitions and facts of fixed point classes of graph selfmaps. In this section, we will state them and define a new homotopy invariant $a(f, \mathbf{F}) \in \mathbb{N}$, called improved characteristic, for any fixed class $\mathbf{F}$ of a $\pi_{1}$-injective selfmap $f: X \rightarrow X$ of a connected finite graph $X$. 
3.1. Fixed point class. Let $p: \tilde{X} \rightarrow X$ be the universal covering of $X$, with group $\pi$ of covering transformations which is identified with the fundamental group $\pi_{1}(X)$.

For any lifting $\tilde{f}: \tilde{X} \rightarrow \tilde{X}$ of $f$, the projection of its fixed point set is called a fixed point class of $f$, written $\mathbf{F}=p($ Fix $\tilde{f})$. Strictly speaking, we say two liftings $\tilde{f}$ and $\tilde{f}^{\prime}$ of $f$ are conjugate if there exists $\gamma \in \pi$ such that $\tilde{f}^{\prime}=\gamma^{-1} \circ \tilde{f} \circ \gamma$. Then $\mathbf{F}=p(\operatorname{Fix} \tilde{f})$ is said to be the fixed point class of $f$ labeled by the conjugacy class of $\tilde{f}$. Thus, a fixed point class always carries a label which is a conjugacy class of liftings. The fixed point set Fix $f$ decomposes into a disjoint union of fixed point classes. When Fix $\tilde{f}=\emptyset$, we call $\mathbf{F}=p(\operatorname{Fix} \tilde{f})$ an empty fixed point class.

Empty fixed point classes have the same index 0 but may have different labels hence be regarded as different. We would better think of them as hidden rather than nonexistent.

3.2. Improved characteristic. Each lifting $\tilde{f}$ induces an injective endomorphism $\tilde{f}_{\pi}$ : $\pi \rightarrow \pi$ defined by

$$
\tilde{f} \circ \gamma=\tilde{f}_{\pi}(\gamma) \circ \tilde{f}, \quad \gamma \in \pi .
$$

If two liftings $\tilde{f}$ and $\tilde{f}^{\prime}$ label the same fixed point class $\mathbf{F}$, i.e., there exists $\gamma \in \pi$ such that $\tilde{f}^{\prime}=\gamma^{-1} \circ \tilde{f} \circ \gamma$, then the induced endomorphism $\tilde{f}_{\pi}^{\prime}=i_{\gamma^{-1}} \circ \tilde{f}_{\pi} \circ i_{\gamma} \in \operatorname{Inj}(\pi)$ and hence the fixed subgroup $\operatorname{Fix}\left(\tilde{f}_{\pi}\right) \cong \operatorname{Fix}\left(\tilde{f}_{\pi}^{\prime}\right)$ and the number $a\left(\tilde{f}_{\pi}\right)=a\left(\tilde{f}_{\pi}^{\prime}\right)$.

The stabilizer of a fixed point class $\mathbf{F}=p($ Fix $\tilde{f})$ is defined as the subgroup

$$
\operatorname{Stab}(f, \mathbf{F}):=\left\{\gamma \in \pi \mid \gamma^{-1} \circ \tilde{f} \circ \gamma=\tilde{f}\right\},
$$

which is identical to the fixed subgroup $\operatorname{Fix}\left(\tilde{f}_{\pi}\right)=\left\{\gamma \in \pi \mid \tilde{f}_{\pi}(\gamma)=\gamma\right\}$. Up to group isomorphism, it is independent of the choice of $\tilde{f}$ in its conjugacy class.

For nonempty fixed point classes, the definitions above reduces to the simpler ones given in Section 1 .

Definition 3.1. Let $f: X \rightarrow X$ be a $\pi_{1}$-injective graph map, and $\mathbf{F}=p($ Fix $\tilde{f})$ be any fixed point class of $f$. Define $\operatorname{rk}(f, \mathbf{F}):=\operatorname{rkStab}(f, \mathbf{F}), a(f, \mathbf{F}):=a\left(\tilde{f}_{\pi}\right)$, and define the improved characteristic of $\mathbf{F}$ to be

$$
\operatorname{ichr}(f, \mathbf{F}):=1-\operatorname{rk}(f, \mathbf{F})-a(f, \mathbf{F}) .
$$

Remark 3.2. Note that $2 \chi(X)-1 \leq \operatorname{ichr}(f, \mathbf{F}) \leq 1$ by Corollary 1.6

By the same argument as the proof of Proposition 2.3 in [GJLL], we have

Proposition 3.3. Let $f: X \rightarrow X$ be a $\pi_{1}$-injective selfmap of a graph. Then for each empty fixed point class $\mathbf{F}$ of $f$, we have $0 \leq \operatorname{ichr}(\mathbf{F}) \leq 1$.

Proof. Let $\mathbf{F}=p($ Fix $\tilde{f})$ be a fixed point class of $f$. By definition, $\operatorname{ichr}(\mathbf{F})=1-\operatorname{rk}(\mathbf{F})-$ $a(\mathbf{F}) \leq 1$. We will show that $\tilde{f}$ has a fixed point when $\operatorname{ichr}(\mathbf{F})<0$.

For the lifting $\tilde{f}: \tilde{X} \rightarrow \tilde{X}$, as in [B], p. 19], we have the following:

Fixed point criterion. Let $x, y \in \tilde{X}$ be two distinct points with the property that $\tilde{f}(x)$ is distinct from $x$ and is not contained in the same connected component of $\tilde{X} \backslash x$ as $y$, and conversely. Then $\tilde{f}$ has a fixed point on the path $[x, y]$. 
Let every edge of $X$ have length 1 . Then $X$ becomes a metric graph, and equip $\tilde{X}$ with the lifted metric. For any point $P \in \tilde{X}$, the map

$$
j: \pi \rightarrow \tilde{X}, \quad w \mapsto w P
$$

gives a quasi-isometric embedding from the covering transformation group $\pi$ to $\tilde{X}$. This induces a homeomorphism between $\partial \pi$ and the space $\partial \tilde{X}$ of ends of $\tilde{X}$, which is independent of the choice $P$. Moreover, the distance between $\tilde{f}(j(w))=\tilde{f}_{\pi}(w) \tilde{f}(P)$ and $j\left(\tilde{f}_{\pi}(w)\right)=\tilde{f}_{\pi}(w) P$ is bounded by the distance between $P$ and $\tilde{f}(P)$, independent of $w \in \pi$. It follows that the extension of $\tilde{f}$ to $\partial \tilde{X}$ agrees with the extension of $\tilde{f}_{\pi}$ to $\partial \pi$. Therefore, an attracting fixed point of $\tilde{f}_{\pi}$ defines an attracting fixed point of $\tilde{f}$ on $\partial \tilde{X}$.

If $\operatorname{ichr}(\mathbf{F})<0$, namely, $\operatorname{rkFix}\left(\tilde{f}_{\pi}\right)+a\left(\tilde{f}_{\pi}\right)>1$ by definition. First assume that $\tilde{f}_{\pi}$ has two distinct (possible equivalent) attracting fixed points $W_{1}, W_{2} \in \partial \pi$. Then any two points $x, y \in \tilde{X}$ sufficiently close to the corresponding attracting fixed points in $\partial \tilde{X}$ satisfy the hypothesis of the above fixed point criterion, and $\tilde{f}$ has a fixed point. Such points $W_{1}, W_{2}$ exist if $a\left(\tilde{f}_{\pi}\right) \geq 2$. They also exist if $a\left(\tilde{f}_{\pi}\right)=1$ while $\operatorname{rkFix}\left(\tilde{f}_{\pi}\right)=$ 1 , namely there exist an attracting fixed point $W_{1} \in \partial \pi$ and a nontrivial element $u \in$ $\operatorname{Fix}\left(\tilde{f}_{\pi}\right)$, because in this case, we can take $W_{2}=u W_{1}$. The only remaining case is when $\operatorname{rkFix}\left(\tilde{f}_{\pi}\right) \geq 2$, then $[\overline{\mathrm{BH}}$, Lemma 2.1] applies.

3.3. Alternative definitions. The above definitions of fixed point class and improved characteristic involve covering spaces. Now we state an alternative approach using paths (introduced in [JWZ, §2]), which is convenient for us to prove the homotopy invariance of $\operatorname{ichr}(\mathbf{F})$.

Definition 3.4. By an $f$-route we mean a homotopy class (rel. endpoints) of path $w: I \rightarrow$ $X$ from a point $x \in X$ to $f(x)$. For brevity, we shall often say the path $w$ (in place of the path class $[w])$ is an $f$-route at $x=w(0)$. An $f$-route $w$ gives rise to an endomorphism

$$
f_{w}: \pi_{1}(X, x) \rightarrow \pi_{1}(X, x),[a] \mapsto[w(f \circ a) \bar{w}]
$$

where $a$ is any loop based at $x$, and $\bar{w}$ denotes the reverse of $w$. For brevity, we will write $f_{\pi}: \pi_{1}(X) \rightarrow \pi_{1}(X)$ when $w$ and the base point $x$ are omitted. Two $f$-routes $[w]$ and $\left[w^{\prime}\right]$ are conjugate if there is a path $q: I \rightarrow X$ from $x=w(0)$ to $x^{\prime}=w^{\prime}(0)$ such that $\left[w^{\prime}\right]=[\bar{q} w(f \circ q)]$, that is, $w^{\prime}$ and $\bar{q} w(f \circ q)$ homotopic rel. endpoints. We also say that the (possibly tightened) $f$-route $\bar{q} w(f \circ q)$ is obtained from $w$ by an $f$-route move along the path $q$.

Note that a constant $f$-route $w$ corresponds to a fixed point $x=w(0)=w(1) \in \operatorname{Fix} f$, and the endomorphism $f_{w}$ becomes the usual

$$
f_{\pi}: \pi_{1}(X, x) \rightarrow \pi_{1}(X, x),[a] \mapsto[f \circ a],
$$

where $a$ is any loop based at $x$. Two constant $f$-routes are conjugate if and only if the corresponding fixed points can be joined by a Nielsen path. This gives the following definition. 
Definition 3.5. With an $f$-route $w$ (more precisely, with its conjugacy class) we associate a fixed point class $\mathbf{F}_{w}$ of $f$, which consists of the fixed points that correspond to constant $f$-routes conjugate to $w$. Thus fixed point classes are associated bijectively with conjugacy classes of $f$-routes. A fixed point class $\mathbf{F}_{w}$ can be empty if there is no constant $f$-route conjugate to $w$. Empty fixed point classes are inessential and distinguished by their associated route conjugacy classes.

Note that this definition is equivalent to the traditional one in Section 3.1 because an $r$-route specifies a lifting $\tilde{f}$. If two $f$-route $w, w^{\prime}$ are associated to the same fixed point class $\mathbf{F}$ of a $\pi_{1}$-injective graph map $f: X \rightarrow X$, then there is a path $q: I \rightarrow X$ from $x=w(0)$ to $x^{\prime}=w^{\prime}(0)$ such that $\left[w^{\prime}\right]=[\bar{q} w(f \circ q)]$. It implies a commutative diagram:

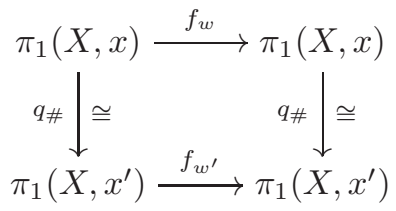

where $q_{\#}: \pi_{1}(X, x) \rightarrow \pi_{1}\left(X, x^{\prime}\right),[a] \mapsto[\bar{q} a q]$ is the induced isomorphism by $q$. Therefore, $\operatorname{rkFix}\left(f_{w}\right)=\operatorname{rkFix}\left(f_{w}^{\prime}\right)$ and $a\left(f_{w}\right)=a\left(f_{w^{\prime}}\right)$, and we can define:

Definition 3.6. For a fixed point class $\mathbf{F}_{w}$ of a $\pi_{1}$-injective graph map $f: X \rightarrow X$, the stabilizer of $\mathbf{F}_{w}$ is defined to be

$$
\operatorname{Stab}\left(f, \mathbf{F}_{w}\right):=\operatorname{Fix}\left(f_{w}\right)=\left\{\gamma \in \pi_{1}(X, w(0)) \mid f_{w}(\gamma)=\gamma\right\},
$$

which is well defined up to isomorphism. Define $\operatorname{rk}\left(f, \mathbf{F}_{w}\right):=\operatorname{rkStab}\left(f, \mathbf{F}_{w}\right), a\left(f, \mathbf{F}_{w}\right):=$ $a\left(f_{w}\right)$, and define the improved characteristic of $\mathbf{F}_{w}$ to be

$$
\operatorname{ichr}\left(f, \mathbf{F}_{w}\right):=1-\operatorname{rk}\left(f, \mathbf{F}_{w}\right)-a\left(f, \mathbf{F}_{w}\right) .
$$

3.4. Invariance. Under a homotopy $H=\left\{h_{t}\right\}_{t \in I}: X \rightarrow X$, each $h_{0}$-route $w_{0}$ gives rise to an $h_{1}$-route $w_{1}=w_{0} \cdot H\left(w_{0}(0)\right)$, where $H\left(w_{0}(0)\right)$ is the path $\left\{h_{t}\left(w_{0}(0)\right)\right\}_{t \in I}$. Clearly $w_{0}$ and $w_{1}$ share the same starting point $w_{0}(0)$, and

$$
\left(h_{0}\right)_{w_{0}}=\left(h_{1}\right)_{w_{1}}: \pi_{1}\left(X, w_{0}(0)\right) \rightarrow \pi_{1}\left(X, w_{0}(0)\right) .
$$

The function $w_{0} \mapsto w_{1}$ defines the fixed point class function $\mathbf{F}_{w_{0}} \mapsto \mathbf{F}_{w_{1}}$ induced by the homotopy. Therefore, when $h_{i}$ is $\pi_{1}$-injective, we have the following fact on the improved characteristic, that is parallel to the one of stabilizer (see [JWZ, §2]).

Fact 1 (Homotopy invariance). A homotopy $H=\left\{h_{t}\right\}_{t \in I}: X \rightarrow X$ gives rise to a bijective correspondence $H: \mathbf{F}_{0} \mapsto \mathbf{F}_{1}$ from $\operatorname{Fpc}\left(h_{0}\right)$ to $\operatorname{Fpc}\left(h_{1}\right)$ with

$$
\operatorname{ind}\left(h_{0}, \mathbf{F}_{0}\right)=\operatorname{ind}\left(h_{1}, \mathbf{F}_{1}\right), \operatorname{Stab}\left(h_{0}, \mathbf{F}_{0}\right) \cong \operatorname{Stab}\left(h_{1}, \mathbf{F}_{1}\right), a\left(h_{0}, \mathbf{F}_{0}\right)=a\left(h_{1}, \mathbf{F}_{1}\right),
$$

which indicate that the index $\operatorname{ind}(\mathbf{F})$ and the improved characteristic ichr $(\mathbf{F})$ are homotopy invariants. 
An inj-morphism from a $\pi_{1}$-injective graph map $f: X \rightarrow X$ to a $\pi_{1}$-injective graph map $g: Y \rightarrow Y$ means a $\pi_{1}$-injective map $h: X \rightarrow Y$ such that $h \circ f=g \circ h$. For an $f$-route $w$ at $x=w(0), h$ induces a commutative diagram:

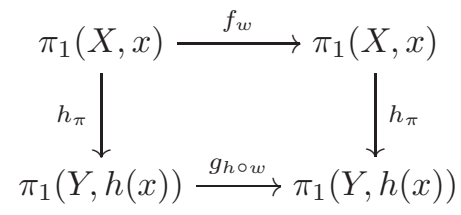

where $h_{\pi}: \pi_{1}(X, x) \rightarrow \pi_{1}(Y, h(x))$ defined by $[a] \mapsto[h \circ a]$ is the injective homomorphism induced by $h$.

Clearly, $\left.h_{\pi}\right|_{\operatorname{Fix}\left(f_{w}\right)}: \operatorname{Fix}\left(f_{w}\right) \rightarrow \operatorname{Fix}\left(g_{h \circ w}\right)$ is injective. For any two attracting fixed words $W \neq V$ of $f_{w}$, by Lemma 2.1, $\bar{h}_{\pi}(W) \neq \bar{h}_{\pi}(V)$ are two attracting fixed words of $g_{h \circ w}$. So $W \mapsto \bar{h}_{\pi}(W)$ defines an injection $\bar{h}_{\pi}: \mathcal{A}\left(f_{w}\right) \hookrightarrow \mathcal{A}\left(g_{h \circ w}\right)$ from the set of attracting fixed words of $f_{w}$ to that of $g_{h \circ w}$.

Fact 2 (Morphism). An inj-morphism $h$ from a $\pi_{1}$-injective selfmap $f$ of graph $X$ to a $\pi_{1}$-injective selfmap $g$ of graph $Y$ induces a natural function $w \mapsto h \circ w$ from $f$-routes to $g$-routes and a function $\mathbf{F}_{w} \mapsto \mathbf{F}_{\text {how }}$ from $\operatorname{Fpc}(f)$ to $\operatorname{Fpc}(g)$ such that $h\left(\mathbf{F}_{w}\right) \subset \mathbf{F}_{h \circ w}$, and two injections

$$
h_{\pi}: \operatorname{Stab}\left(f, \mathbf{F}_{w}\right) \hookrightarrow \operatorname{Stab}\left(g, \mathbf{F}_{h \circ w}\right), \quad \bar{h}_{\pi}: \mathcal{A}\left(f_{w}\right) \hookrightarrow \mathcal{A}\left(g_{h \circ w}\right) .
$$

Furthermore, when $\left.h_{\pi}\right|_{\operatorname{Fix}\left(f_{w}\right)}: \operatorname{Fix}\left(f_{w}\right) \cong \operatorname{Fix}\left(g_{h \circ w}\right)$, two attracting fixed words $W, V \in \mathcal{A}\left(f_{w}\right)$ are equivalent if and only if their images $\bar{h}_{\pi}(W), \bar{h}_{\pi}(V) \in \mathcal{A}\left(g_{h \circ w}\right)$ are equivalent, namely, there exists a word $\omega \in \operatorname{Fix}\left(g_{h \circ w}\right)$ such that $\bar{h}_{\pi}(W)=\omega \bar{h}_{\pi}(V)$ if and only if $W=h_{\pi}^{-1}(\omega) V$. It implies an injection $\bar{h}_{\pi}: \mathscr{A}\left(f_{w}\right) \hookrightarrow \mathscr{A}\left(g_{h \circ w}\right)$ defined by $[W] \mapsto\left[\bar{h}_{\pi}(W)\right]$, where $[W]$ denotes the equivalence class represented by $W$. Therefore

Fact 3 (Commutation invariance). Suppose $\phi: X \rightarrow Y$ and $\psi: Y \rightarrow X$ are $\pi_{1}$-injective graph maps. Then the $\pi_{1}$-injective selfmap $\psi \circ \phi: X \rightarrow X$ and $\phi \circ \psi: Y \rightarrow Y$ are said to differ by a commutation. The inj-morphism $\phi$ between them sets up a natural bijective correspondence $\mathbf{F}_{\psi \circ \phi} \mapsto \mathbf{F}_{\phi \circ \psi}$ from $\operatorname{Fpc}(\psi \circ \phi)$ to $\operatorname{Fpc}(\phi \circ \psi)$, with

$$
\operatorname{ind}\left(\psi \circ \phi, \mathbf{F}_{\psi \circ \phi}\right)=\operatorname{ind}\left(\phi \circ \psi, \mathbf{F}_{\phi \circ \psi}\right),
$$

and

$$
\operatorname{Stab}\left(\psi \circ \phi, \mathbf{F}_{\psi \circ \phi}\right) \cong \operatorname{Stab}\left(\phi \circ \psi, \mathbf{F}_{\phi \circ \psi}\right), \quad a\left(\psi \circ \phi, \mathbf{F}_{\psi \circ \phi}\right)=a\left(\phi \circ \psi, \mathbf{F}_{\phi \circ \psi}\right)
$$

which indicate that $\operatorname{ind}(\mathbf{F})$ and $\operatorname{ichr}(\mathbf{F})$ are commutation invariants.

Remark 3.7. A homotopy may create nonempty fixed point classes, or remove fixed point classes. The above correspondence is bijective only when empty fixed point classes are taken into account. 
Example 3.8. Let $f: S^{1} \rightarrow S^{1}$ be a $\pi_{1}$-injective selfmap of the circle. Then $f$ can be homotoped to a map $g: S^{1} \rightarrow S^{1}$ defined by $g\left(e^{i \theta}\right)=e^{i k \theta}$, where $k \neq 0$ is the degree of $f$. Note that all fixed point classes of $g$ induce the same homomorphism $g_{\pi}(z)=z^{k}$ where $z$ is a generator of the fundamental group $\pi_{1}\left(S^{1}\right)=\mathbb{Z}$.

If $k=1$, then $g$ is the identity and every fixed point class $\mathbf{F}$ of $g$ is inessential, i.e., $\operatorname{ind}(\mathbf{F})=0$. From Example 2.7, we have that $\operatorname{rk}(\mathbf{F})=1, a(\mathbf{F})=0$ and hence

$$
\operatorname{ind}(\mathbf{F})=\operatorname{ichr}(\mathbf{F})=1-\operatorname{rk}(\mathbf{F})-a(\mathbf{F})=0 .
$$

If $k \neq 1$, then every lifting of $g$ has a fixed point and hence every fixed point class $\mathbf{F}$ of $g$ consists of a single point. It is well-known that all these fixed point classes have the same index ind $(\mathbf{F})=\operatorname{sgn}(1-k)$. From Example 2.7, we also have ind $(\mathbf{F})=\operatorname{ichr}(\mathbf{F})$.

So, by the homotopy invariance, we have

$$
\operatorname{ind}(\mathbf{F})=\operatorname{ichr}(\mathbf{F})
$$

for every fixed point class $\mathbf{F}$ of $f: S^{1} \rightarrow S^{1}$.

\section{GRAPH MAPS}

In this section, we will study properties of the improved characteristic of graph maps. Since our discussion of graph maps is based on Bestvina and Handel's theory of train tracks $[\mathrm{BH}]$. We follow their terminology first.

4.1. Relative train track map. A graph $X$ is a 1-dimensional (or possibly 0-dimensional) finite cellular complex. The 0 -cells and (open) 1-cells are called vertices and edges respectively. A graph map $f: X \rightarrow Y$ is a cellular map, that is, it maps vertices to vertices. Up to homotopy there is no loss to assume that the restriction of $f$ to every edge $e$ of $X$ is either locally injective or equal to a constant map. A graph map $f: X \rightarrow Y$ is $\pi_{1}$-injective if it induces an injective homomorphism of the fundamental group on each component of $X$. It is an immersion if it sends edges to edges and it is locally injective at vertices. Clearly immersions are always $\pi_{1}$-injective.

A path $p$ in a graph $X$ is a map $p:[0,1] \rightarrow X$ that is either locally injective or equal to a constant map, in the latter case we say that $p$ is a trivial path. Combinatorially, a nontrivial path consists of a finite sequence $e_{1}, e_{2}, \ldots, e_{k}$ of oriented edges $e_{i}$ with consecutive edges $e_{i}, e_{i+1}$ adjacent but $e_{i+1}$ not the inverse $\bar{e}_{i}$ of $e_{i}$. For a nontrivial path $p$ in $X$, its initial tip is the maximal initial open subpath that lies in an edge of $X$. The terminal tip is defined similarly.

A ray $\rho$ in a connected graph $X$ (equipping each edge with length 1 ) is a locally isometric map $\rho:[0,+\infty) \rightarrow X$, or combinatorially, a ray consists of an infinite sequence $e_{1}, e_{2}, \ldots$ of oriented edges $e_{i}$ with consecutive edges $e_{i}, e_{i+1}$ adjacent but $e_{i+1}$ not the inverse of $e_{i}$. The point $\rho(0)$ is called the origin of $\rho$. If fixing a universal covering $q: \tilde{X} \rightarrow X$ and $\tilde{v} \in q^{-1}(\rho(0))$, then a lifting $\tilde{\rho}:[0,+\infty) \rightarrow \tilde{X}$ with origin $\tilde{\rho}(0)=\tilde{v}$ is an isometric embedding, and its image looks like a real ray in the tree $\tilde{X}$. A sub-ray of a ray $\tilde{\rho}$ means a restriction $\left.\tilde{\rho}\right|_{[n,+\infty]}$. For any two points $\tilde{x}, \tilde{y} \in \tilde{X}$, let $[\tilde{x}, \tilde{y}]$ denote the 
unique path from $\tilde{x}$ to $\tilde{y}$ in the tree $\tilde{X}$. Two rays (with different origins) are equivalent if their intersection has infinite length. In particular, a ray is always equivalent to its sub-rays. Each equivalence class of rays in $\tilde{X}$ defines an end of the tree $\tilde{X}$. Let $\partial \tilde{X}$ denote the space of all the ends of $\tilde{X}$. Note that there is a bijective correspondence between the space $\partial \tilde{X}$ and the set of rays in $\tilde{X}$ with the same origin. For brevity, we often identify a ray or a path with its image.

A turn in $X$ is an unordered pair of oriented edges of $X$ originating at a common vertex. A turn is nondegenerate if it is defined by distinct oriented edges, and is degenerate otherwise. A graph map $f: X \rightarrow Y$ induces a function $D f$ on the set $\mathrm{E}(X)$ of oriented edges of $X$ by sending an oriented edge $e$ to the first oriented edge of $f(e)$; if $f(e)$ is trivial we say $D f(e)=0$. A turn $\left\{e_{1}, e_{2}\right\}$ is illegal with respect to $f: X \rightarrow Y$ if the image $\left\{D f^{k}\left(e_{1}\right), D f^{k}\left(e_{2}\right)\right\}$ under some iterate of $D f$ is degenerate, and is legal otherwise.

For a selfmap $f: X \rightarrow X$ of graph $X$, an $f$-Nielsen path is a nontrivial path $p$ in $X$ joining two fixed points of $f$ such that $f \circ p \simeq p$ rel. endpoints; it is indivisible if it cannot be written as a concatenation $p=p_{1} \cdot p_{2}$, where $p_{1}$ and $p_{2}$ are subpaths of $p$ that are $f$-Nielsen paths.

Add [BH, Lemma 5.11] or [DV, Proposition IV 3.2] to [JWZ, Theorem BH], we have the following theorem summarizing the results of Bestvina and Handel [BH] that we need.

Theorem 4.1. [JWZ, Theorem $\mathrm{BH}]$ Let $X$ be a connected graph but not a tree, and let $f: X \rightarrow X$ be a $\pi_{1}$-injective map. Then $f$ has the same homotopy type as a graph selfmap $\beta: Z \rightarrow Z$, where $Z$ is a connected graph without vertices of valence 1 and all fixed points of $\beta$ are vertices, and there is a $\beta$-invariant proper subgraph $Z_{0}$, containing all vertices of $Z$. The map $\beta:\left(Z, Z_{0}\right) \rightarrow\left(Z, Z_{0}\right)$ of the pair is of one of the following types.

Type $1: \beta$ sends $Z$ into $Z_{0}$.

Type $2: \beta$ cyclically permutes the edges in $Z \backslash Z_{0}$.

Type $3: \beta$ expands edges of $Z \backslash Z_{0}$ by a factor $\lambda>1$ with respect to a suitable nonnegative metric $L$ supported on $Z \backslash Z_{0}$, and has the properties (a)-(c) below.

(a) For every oriented edge e in $Z \backslash Z_{0}, D \beta(e)$ lies in $Z \backslash Z_{0}$.

(b) There is at most one indivisible $\beta$-Nielsen path that intersects $Z \backslash Z_{0}$.

(c) If $p$ is an indivisible $\beta$-Nielsen path that intersects $Z \backslash Z_{0}$, then $p=p_{1} \bar{p}_{2}$, where $p_{1}, p_{2}$ are $\beta$-legal paths with length $L\left(p_{1}\right)=L\left(p_{2}\right)$, and the turn $\left\{\bar{p}_{1}, \bar{p}_{2}\right\}$ is the unique illegal turn in $Z \backslash Z_{0}$ (at a vertex $v_{p}=p_{1}(1)=p_{2}(1)$ of valence $\geq 3$ in $Z$ ) which degenerates under $D \beta$. Moreover, $\beta\left(p_{i}\right)=p_{i} t(i=1,2)$ where $t$ is a $\beta$-legal path (see Figure $\square$ below).

A graph map as in Theorem 4.1 is called a relative train track map, for short, say an RTT map.

Let $\beta:\left(Z, Z_{0}\right) \rightarrow\left(Z, Z_{0}\right)$ be an RTT map. Denote $\beta_{0}:=\left.\beta\right|_{Z_{0}}: Z_{0} \rightarrow Z_{0}$. In order to discuss the index and the improved characteristic, we introduce some notations.

Let $\mathrm{V}(Z)$ be the set of vertices of $Z$, and $\mathrm{E}\left(Z \backslash Z_{0}\right)$ the set of oriented edges of $Z \backslash Z_{0}$. For a fixed vertex $v \in \mathrm{V}(Z)$, let $\delta(v)$ be the number of oriented edges $e \in \mathrm{E}\left(Z \backslash Z_{0}\right)$ with 


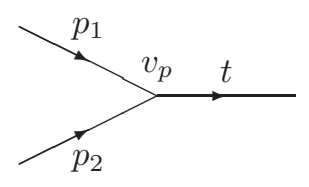

FIGURE 1. An indivisible Nielsen path.

the additional requirement that $e$ gets initially expanded along itself by $\beta$ (In Type 2, when $Z \backslash Z_{0}$ is a single edge $e$, consider that $e$ gets initially expanded along itself on one tip and shrinks on the other), that is

$$
\Delta(v):=\left\{e \in \mathrm{E}\left(Z \backslash Z_{0}\right) \mid e(0)=v, D \beta(e)=e\right\}, \quad \delta(v):=\# \Delta(v) .
$$

For a nonempty fixed point class $\mathbf{F}$ of $\beta$, let

$$
\Delta(\mathbf{F}):=\left\{e \in \mathrm{E}\left(Z \backslash Z_{0}\right) \mid e(0) \in \mathbf{F}, D \beta(e)=e\right\}=\bigsqcup_{v \in \mathbf{F}} \Delta(v),
$$

and let $\delta(\mathbf{F}):=\# \Delta(\mathbf{F})=\sum_{v \in \mathbf{F}} \delta(v)$. Recall that $\operatorname{ind}(\beta, v)=\operatorname{ind}\left(\beta_{0}, v\right)-\delta(v)$ for any fixed point $v$ of $\beta_{0}$. So, by the additivity of index, for every $\beta$-fixed point class $\mathbf{F}$, we have

$$
\operatorname{ind}(\beta, \mathbf{F})=\operatorname{ind}\left(\beta_{0}, \mathbf{F}\right)-\delta(\mathbf{F}),
$$

where $\operatorname{ind}\left(\beta_{0}, \mathbf{F}\right)=\sum_{i=1}^{k} \operatorname{ind}\left(\beta_{0}, \mathbf{F}_{i}\right)$ if $\mathbf{F}=\sqcup \mathbf{F}_{i}$ is a union of finite many $\beta_{0}$-fixed point classes $\mathbf{F}_{i}, i=1, \ldots, k$.

4.2. A bijective correspondence. For any nonempty fixed point class $\mathbf{F}$ of $\beta: Z \rightarrow Z$, to give some information on $a(\beta, \mathbf{F})$, we fix a universal covering $q: \tilde{Z} \rightarrow \tilde{Z}$ of $Z$, with group $\pi$ of covering transformations identified with the fundamental group $\pi_{1}\left(Z, v_{0}\right)$ for $v_{0} \in \mathbf{F}$. Pick $\tilde{v}_{0} \in q^{-1}\left(v_{0}\right)$ and a lifting $\tilde{\beta}: \tilde{Z} \rightarrow \tilde{Z}$ of $\beta$ with $\tilde{\beta}\left(\tilde{v}_{0}\right)=\tilde{v}_{0}$, then $\mathbf{F}=q(\operatorname{Fix} \tilde{\beta})$, and the lifting $\tilde{\beta}$ induces an injective endomorphism $\beta_{\pi}: \pi \rightarrow \pi$ defined by

$$
\tilde{\beta} \circ \gamma=\beta_{\pi}(\gamma) \circ \tilde{\beta}, \quad \forall \gamma \in \pi .
$$

Endow $\tilde{Z}$ with a metric $d$ with each edge length 1 . Then the map

$$
j: \pi \rightarrow \tilde{Z}, \quad \gamma \mapsto \gamma\left(\tilde{v}_{0}\right)
$$

is $\pi$-equivariant (i.e. $\alpha(j(\gamma))=j(\alpha \gamma)$ for any $\alpha, \gamma \in \pi$ ), and gives a quasi-isometric embedding from the covering transformation group $\pi$ to the covering space $\tilde{Z}$. This induces a $\pi$-equivariant homeomorphism $\bar{j}: \partial \pi \rightarrow \partial \tilde{Z}$ between $\partial \pi$ and the space $\partial \tilde{Z}$ of ends of $\tilde{Z}$. Moreover, since $\tilde{\beta}(j(\gamma))=\beta_{\pi}(\gamma)\left(\tilde{\beta}\left(\tilde{v}_{0}\right)\right)=\beta_{\pi}(\gamma)\left(\tilde{v}_{0}\right)=j\left(\beta_{\pi}(\gamma)\right)$, we have a 
commutative diagram

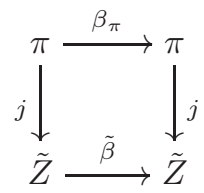

It follows that the extension of $\tilde{\beta}$ to $\partial \tilde{Z}$ agrees with the extension of $\beta_{\pi}$ to $\partial \pi$. Therefore, we have

Assertion $\star$. An attracting fixed word $W \in \partial \pi$ of $\beta_{\pi}$ defines an attracting fixed point $\bar{j}(W) \in \partial \tilde{Z}$ of $\tilde{\beta}$, and the $\pi$-equivariant homeomorphism $\bar{j}: \partial \pi \rightarrow \partial \tilde{Z}$ induces a bijective correspondence

$$
\left.\bar{j}\right|_{\mathcal{A}\left(\beta_{\pi}\right)}: \mathcal{A}\left(\beta_{\pi}\right) \rightarrow \mathcal{A}(\tilde{\beta})
$$

between the set $\mathcal{A}\left(\beta_{\pi}\right)$ of attracting fixed words of $\beta_{\pi}$ in $\partial \pi$ and the set $\mathcal{A}(\tilde{\beta})$ of attracting fixed points of $\tilde{\beta}$ in $\partial \tilde{Z}$.

Here a fixed end $\mathcal{E} \in \mathcal{A}(\tilde{\beta})$ represented by a ray $\tilde{\rho}=\tilde{e}_{1} \cdots \tilde{e}_{i} \cdots \subset \tilde{Z}$ is an attracting fixed point of $\tilde{\beta}$, if there exists a number $N>0$ such that for any point $\tilde{x} \in \tilde{Z}$, we have

$$
d\left(\left[\tilde{v}_{0}, \tilde{x}\right] \cap \tilde{\rho}\right)>N \Longrightarrow \lim _{k \rightarrow+\infty} d\left(\left[\tilde{v}_{0}, \tilde{\beta}^{k}(\tilde{x})\right] \cap \tilde{\rho}\right)=+\infty
$$

that is the same as the one in free groups (see Section 2.1.

4.3. RTT map of Type 3. In this subsection, let $\beta:\left(Z, Z_{0}\right) \rightarrow\left(Z, Z_{0}\right)$ be an RTT map of Type 3 in Theorem 4.1

A path with no illegal turns in $Z \backslash Z_{0}$ is said to be $\beta$-legal. Under the non-negative metric $L$ supported on $Z \backslash Z_{0}$, we have $L(\beta(\sigma))=\lambda L(\sigma)$ for any $\beta$-legal path $\sigma$. For convenience, we state [BH, Lemma 5.8] as follows:

Lemma 4.2. Suppose $\sigma=\mu_{1} \tau_{1} \mu_{2} \ldots \tau_{l-1} \mu_{l}$ is a decomposition of a $\beta$-legal path $\sigma$ into subpaths $\mu_{j} \subset Z \backslash Z_{0}$ and $\tau_{j} \subset Z_{0}$. Then

$$
[\beta(\sigma)]=\beta\left(\mu_{1}\right) \cdot\left[\beta\left(\tau_{1}\right)\right] \cdot \beta\left(\mu_{2}\right) \cdots\left[\beta\left(\tau_{l-1}\right)\right] \cdot \beta\left(\mu_{l}\right)
$$

and is $\beta$-legal. Here $[\beta(\sigma)]$ denotes the path tightened from $\beta(\sigma)$, and $\beta\left(\mu_{i}\right) \cdot\left[\beta\left(\tau_{i}\right)\right]$ indicates that the turns between $\beta\left(\mu_{i}\right)$ and $\left[\beta\left(\tau_{i}\right)\right]$ are legal.

In the following, we shall give a key lemma that summarizes the relation between $\beta$ invariant edges and attracting fixed words of $\beta_{\pi}$.

Lemma 4.3. Let $\beta:\left(Z, Z_{0}\right) \rightarrow\left(Z, Z_{0}\right)$ be an RTT map of Type 3, and $\mathbf{F}$ a nonempty fixed class of $\beta$. Suppose $v_{0} \in \mathbf{F} \subset \mathrm{V}(Z)$ is a fixed point of $\beta$, and $\beta_{\pi}: \pi_{1}\left(Z, v_{0}\right) \rightarrow \pi_{1}\left(Z, v_{0}\right)$ is the induced injective endomorphism of $\beta$. Then

(1) Every oriented edge $e \in \Delta(\mathbf{F})$ defines an equivalence class $\mathscr{W}_{e}$ of attracting fixed words of $\beta_{\pi}$, and $\mathscr{W}_{e}$ does not contain any attracting fixed word of $\left(\beta_{0}\right)_{\pi}: \pi_{1}\left(Z_{0}, v_{0}\right) \rightarrow$ $\pi_{1}\left(Z_{0}, v_{0}\right)$.

(2) Suppose $e_{i} \in \Delta(\mathbf{F})(i=1,2)$ are two edges with initial points $e_{i}(0)$ two (possibly the same) fixed points in $\mathbf{F}$. Then $\mathscr{W}_{e_{1}}$ and $\mathscr{W}_{e_{2}}$ are equal if and only if there exists an 
indivisible Nielsen path $p=p_{1} \bar{p}_{2}$ as in Type 3(c) of Theorem 4.1 such that $e_{i}=D \beta\left(p_{i}\right)$ are the initial edges of the $\beta$-legal paths $p_{i}$ for $i=1,2$.

(3) For every equivalence class $\mathscr{W}$ of attracting fixed words of $\beta_{\pi}$ not containing an attracting fixed word of $\left(\beta_{0}\right)_{\pi}$, there exists an oriented edge e $\in \Delta(\mathbf{F})$ such that $\mathscr{W}=\mathscr{W}_{e}$.

Proof. Pick a universal covering $q: \tilde{Z} \rightarrow \tilde{Z}$ and a lifting $\tilde{\beta}: \tilde{Z} \rightarrow \tilde{Z}$ as in Section 4.2 Let $\tilde{Z}_{0}:=q^{-1}\left(Z_{0}\right)$.

(1) For any edge $e \in \Delta(\mathbf{F})$, let $v:=e(0) \in \mathbf{F}$. Recall that there is a non-negative metric $L$ supported on $Z \backslash Z_{0}$, such that $\beta$ expands $e$ by a factor $\lambda>1$ with respect to $L$, namely, $L(\beta(e))=\lambda L(e)$, which implies that $\beta(e)$ passes at least two edges of $Z \backslash Z_{0}$. Property (a) in Theorem 4.1 implies both tips of $\beta(e)$ are in $Z \backslash Z_{0}$. Furthermore, we have a decomposition of $\beta(e)$ as in Lemma 4.2 $\beta(e)=e \cdot \tau_{1} \cdot \mu_{2} \cdots \tau_{l-1} \cdot \mu_{l}$ is a $\beta$-legal path, and

$$
\begin{aligned}
{\left[\beta^{2}(e)\right] } & =\beta(e) \cdot\left[\beta\left(\tau_{1}\right)\right] \cdot \beta\left(\mu_{2}\right) \cdots\left[\beta\left(\tau_{l-1}\right)\right] \cdot \beta\left(\mu_{l}\right) \\
& =e \cdot \tau_{1} \cdot \mu_{2} \cdots \tau_{l-1} \cdot \mu_{l} \cdot\left[\beta\left(\tau_{1}\right)\right] \cdot \beta\left(\mu_{2}\right) \cdots\left[\beta\left(\tau_{l-1}\right)\right] \cdot \beta\left(\mu_{l}\right) .
\end{aligned}
$$

By induction, the common initial segment $\left[\beta^{k}(e)\right] \cap\left[\beta^{k+1}(e)\right]=\left[\beta^{k}(e)\right]$ for any $k \in \mathbb{N}$. Therefore, we can define $\beta^{\infty}(e):=\lim _{k \rightarrow+\infty}\left[\beta^{k}(e)\right]$. Note that $\beta\left(\beta^{\infty}(e)\right)=\beta^{\infty}(e)$, so it is a $\beta$-invariant ray in $Z$.

Below we will lift $\beta^{\infty}(e)$ to the universal covering $q: \tilde{Z} \rightarrow \tilde{Z}$, and then show that the lifting is an attracting fixed point of $\tilde{\beta}$.

Recall that $\tilde{\beta}: \tilde{Z} \rightarrow \tilde{Z}$ is a lifting of $\beta$ such that $\tilde{\beta}\left(\tilde{v}_{0}\right)=\tilde{v}_{0}$. Since $v$ and $v_{0}$ are in the same fixed point class $\mathbf{F}$, we have $q^{-1}(v) \cap \operatorname{Fix} \tilde{\beta} \neq \emptyset$. Pick a lifting $\tilde{v} \in q^{-1}(v) \cap \operatorname{Fix} \tilde{\beta}$ and a lifting $\tilde{e}$ of $e$ with $\tilde{e}(0)=\tilde{v}$. Then we have a $\tilde{\beta}$-invariant ray

$$
\tilde{\beta}^{\infty}(\tilde{e}):=\lim _{k \rightarrow+\infty}\left[\tilde{\beta}^{k}(\tilde{e})\right]=\bigcup_{k \rightarrow+\infty}\left[\tilde{\beta}^{k}(\tilde{e})\right]:[0,+\infty] \rightarrow \tilde{Z}
$$

which is a lifting of $\beta^{\infty}(e)$, with origin $\tilde{v}$.

Endow $\tilde{Z}$ with the lifted metric of $L$ (still written $L$ ), we have $L\left(\left[\tilde{\beta}^{k}(\tilde{e})\right]\right)=\lambda^{k} L(\tilde{e})$. If we equip $\tilde{Z}$ with the natural metric $d$ with each edge length 1 , then we have

$$
d\left(\left[\tilde{\beta}^{k}(\tilde{e})\right]\right) \geq L\left(\left[\tilde{\beta}^{k}(\tilde{e})\right]\right) / l_{0}=\lambda^{k} L(\tilde{e}) / l_{0}
$$

where $l_{0}:=\max \{L(e) \mid e \in \mathrm{E}(Z)\}$ is finite because $Z$ is a finite graph. So the "bounded cancellation lemma" $([\overline{\mathrm{C}}]$ or $[\overline{\mathrm{DV}}])$ implies that there exists $N>0$ such that for any point $\tilde{x} \in \tilde{Z}$,

$$
d\left(\left[\tilde{v}_{0}, \tilde{x}\right] \cap \tilde{\beta}^{\infty}(\tilde{e})\right)>N \Longrightarrow \lim _{k \rightarrow+\infty} d\left(\left[\tilde{v}_{0}, \tilde{\beta}^{k}(\tilde{x})\right] \cap \tilde{\beta}^{\infty}(\tilde{e})\right)=+\infty,
$$

i.e., the end represented by the $\tilde{\beta}$-invariant ray $\tilde{\beta}^{\infty}(\tilde{e})$ is an attracting fixed point of $\tilde{\beta}$ on the space $\partial \tilde{Z}$. By Assertion $\star$, it defines an attracting fixed word $W_{\tilde{e}}$ of $\beta_{\pi}$, that is, $\bar{j}\left(W_{\tilde{e}}\right)=\tilde{\beta}^{\infty}(\tilde{e})$.

If $\tilde{e}^{\prime}$ is another lifting of $e$ with origin $\tilde{v}^{\prime} \in q^{-1}(v) \cap \operatorname{Fix} \tilde{\beta}$, there exists $\omega \in \pi$ such that $\omega(\tilde{v})=\tilde{v}^{\prime}$. It implies $\omega \in \operatorname{Fix}\left(\beta_{\pi}\right)$ and the ray $\tilde{\beta}^{\infty}\left(\tilde{e}^{\prime}\right)=\omega\left(\tilde{\beta}^{\infty}(\tilde{e})\right)$. So the attracting 
fixed point $W_{\tilde{e}^{\prime}}=\omega W_{\tilde{e}}$, namely, $W_{\tilde{e}^{\prime}}$ and $W_{\tilde{e}}$ are equivalent and hence represent the same equivalence class of attracting fixed points of $\beta_{\pi}$, which is denoted by $\mathscr{W}_{e}$.

If $U$ is an attracting fixed word of $\left(\beta_{0}\right)_{\pi}$, then the ray $\bar{j}(U) \subset q^{-1}\left(Z_{0}\right)$ and has length $L(\bar{j}(U))=0$, but any ray representing $\mathscr{W}_{e}$ has length $L=\infty$. So the equivalence class $\mathscr{W}_{e}$ can not contain any attracting fixed word of $\left(\beta_{0}\right)_{\pi}$.

(2) For two edges $e_{i} \in \Delta(\mathbf{F})(i=1,2)$ with initial points $e_{i}(0)$ two (possibly the same) fixed points in $\mathbf{F}$, if there exists an indivisible Nielsen path $p=p_{1} \bar{p}_{2}$ as in Type 3(c) of Theorem 4.1 such that $e_{i}=D \beta\left(p_{i}\right)$ for $i=1,2$, by lifting $p$ to the universal covering $\tilde{Z}$, we have an indivisible Nielsen path $\tilde{p}=\tilde{p}_{1} \tilde{\bar{p}}_{2}$ of $\tilde{\beta}$ with tips $D \tilde{\beta}\left(\tilde{p}_{i}\right)=\tilde{e}_{i}$ that is a lifting of $e_{i}$. Moreover, since $\tilde{\beta}\left(\tilde{p}_{i}\right)=\tilde{p}_{i} \tilde{t}$ for $\tilde{t}$ a $\tilde{\beta}$-legal path, the intersection of the rays $\tilde{\beta}^{\infty}\left(\tilde{e}_{1}\right)=\tilde{\beta}^{\infty}\left(\tilde{p}_{1}\right)$ and $\tilde{\beta}^{\infty}\left(\tilde{e}_{2}\right)=\tilde{\beta}^{\infty}\left(\tilde{p}_{2}\right)$ have infinite length, which implies that they represent the same end of $\tilde{Z}$ and hence the same attracting fixed point of $\tilde{\beta}$. Therefore, by the above Assertion $\star, e_{1}$ and $e_{2}$ define the same equivalence class $\mathscr{W}_{e_{1}}=\mathscr{W}_{e_{2}}$ of attracting fixed words of $\beta_{\pi}$.

Conversely, if the two equivalence classes $\mathscr{W}_{e_{1}}$ and $\mathscr{W}_{e_{2}}$ of attracting fixed words of $\beta_{\pi}$ are the same, there exists lifting $\tilde{e}_{i}$ of $e_{i}$ such that $W_{\tilde{e}_{1}}=W_{\tilde{e}_{2}}$. It implies that the intersection $\tilde{\rho}:=\tilde{\beta}^{\infty}\left(\tilde{e}_{1}\right) \cap \tilde{\beta}^{\infty}\left(\tilde{e}_{2}\right)$ of the two $\tilde{\beta}$-invariant rays $\tilde{\beta}^{\infty}\left(\tilde{e}_{i}\right)$ has length $L(\tilde{\rho})=\infty$. So we can denote $\tilde{\beta}^{\infty}\left(\tilde{e}_{i}\right)=\tilde{p}_{i} \tilde{\rho}$, where $\tilde{p}_{i}$ is a $\tilde{\beta}$-legal path with initial edge $\tilde{e}_{i}$, and the turn $\left\{\overline{\tilde{p}}_{1}, \overline{\tilde{p}}_{2}\right\}$ is nondegenerate, but the turn $\left\{D \tilde{\beta}\left(\overline{\tilde{p}}_{1}\right), D \tilde{\beta}\left(\overline{\tilde{p}}_{2}\right)\right\}$ is degenerate. So $\tilde{p}_{1} \overline{\tilde{p}}_{2}$ equals the tightened path $\left[\tilde{\beta}\left(\tilde{p}_{1} \overline{\tilde{p}}_{2}\right)\right]$ and hence is a Nielsen path of $\tilde{\beta}$. Furthermore, since $\tilde{\beta}$ expands $\tilde{p}_{i}$ by the factor $\lambda>1$ w.r.t the metric $L$, the initial point $\tilde{p}_{i}(0)$ is the unique fixed point in $\tilde{p}_{i}$. It follows that the Nielsen path $\tilde{p}_{1} \overline{\tilde{p}}_{2}$ is indivisible, and hence $p_{1} \bar{p}_{2}:=q\left(\tilde{p}_{1} \overline{\tilde{p}}_{2}\right)$ is an indivisible Nielsen path satisfying the property in claim (2).

(3) For any equivalence class $\mathscr{W}$ of attracting fixed words with property as in claim (3), by the above Assertion $\star$, there exists $W \in \mathscr{W}$ such that $\bar{j}(W) \in \partial \tilde{Z}$ is an attracting fixed point of $\tilde{\beta}$. Suppose $\rho:[0,+\infty] \rightarrow \tilde{Z}$ is a ray representing $\bar{j}(W)$ with origin $\rho(0)=\tilde{v}_{0}$ (see Figure 2 below). Since $\bar{j}(W)$ is attracting (under the metric $d$ on $\tilde{Z}$ that every edge has length 1), the set $S_{\rho}:=\rho \cap \operatorname{Fix} \tilde{\beta}$ of $\tilde{\beta}$-fixed points in $\rho$ is finite. Indeed, if $S_{\rho}$ is infinite, there exists an infinite sequence $\tilde{v}_{i} \in S_{\rho}$ such that the length $d\left(\left[\tilde{v}_{0}, \tilde{v}_{i}\right]\right)$ of $\tilde{\beta}$-Nielsen path goes to infinity as $i \rightarrow+\infty$, but for any given $\tilde{v}_{i} \in S_{\rho}, d\left(\left[\tilde{v}_{0}, \tilde{\beta}^{k}\left(\tilde{v}_{i}\right)\right] \cap \rho\right)=d\left(\left[\tilde{v}_{0}, \tilde{v}_{i}\right]\right)$ does not go to infinity as $k \rightarrow+\infty$, contradicting that $\rho$ is attracting.

Let $\tilde{v}^{\prime}$ be the last one in $S_{\rho}$, that is

$$
d\left(\left[\tilde{v}_{0}, \tilde{v}^{\prime}\right]\right)=\max \left\{d\left(\left[\tilde{v}_{0}, \tilde{v}_{i}\right]\right) \mid \tilde{v}_{i} \in S_{\rho}\right\} .
$$

Let $\rho^{\prime}$ denote the sub-ray of $\rho$ with fixed origin $\rho^{\prime}(0)=\tilde{v}^{\prime}$, and let $\tilde{e}$ be the initial oriented edge of $\rho^{\prime}$ with $\tilde{e}(0)=\tilde{v}^{\prime}$. Note that $\rho^{\prime}$ also represents the equivalence class $\mathscr{W}$ of attracting fixed points of $\tilde{\beta}$. In the tree $\tilde{Z}$, if $D \tilde{\beta}(\tilde{e}) \neq \tilde{e}$, then any two points $x, y \in \rho^{\prime}$ sufficiently close to $\tilde{v}^{\prime}$ and $\partial \tilde{Z}$ respectively satisfy the hypothesis of the fixed point criterion (see the proof of Proposition 3.3), and hence $\tilde{\beta}$ has a fixed point in the path $[x, y] \subset \rho^{\prime}$, that is, $\tilde{\beta}$ 
has another fixed point but not $\tilde{v}^{\prime}$ in $\rho^{\prime}$, contradicting with the choice of $\tilde{v}^{\prime}$. Therefore, we have $D \tilde{\beta}(\tilde{e})=\tilde{e}$ and $\left[\tilde{\beta}\left(\rho^{\prime}\right)\right]=\rho^{\prime}$.

Below we will show that $\tilde{e} \subset \tilde{Z} \backslash \tilde{Z}_{0}$.

Recall that the equivalence class $\mathscr{W}$ represented by $\rho^{\prime}$ does not contain any fixed word in $\partial \pi_{1}\left(Z_{0}\right)$, then $\rho^{\prime}$ contains at least one edge in $\tilde{Z} \backslash \tilde{Z}_{0}$. Let $\tilde{e}^{\prime} \in \mathrm{E}\left(\tilde{Z} \backslash \tilde{Z}_{0}\right)$ be the first one along $\rho^{\prime}$ from its origin $\tilde{v}^{\prime}$, that is

$$
d\left(\left[\tilde{v}^{\prime}, \tilde{e}^{\prime}(0)\right]\right):=\min \left\{d\left(\left[\tilde{v}^{\prime}, \tilde{e}_{i}(0)\right]\right) \mid \tilde{e}_{i} \in \rho^{\prime} \cap \mathrm{E}\left(\tilde{Z} \backslash \tilde{Z}_{0}\right)\right\} .
$$

It follows that the (possibly trivial) path $\rho_{1}:=\left[\tilde{v}^{\prime}, \tilde{e}^{\prime}(0)\right] \subset \tilde{Z}_{0}$, and $\tilde{e}^{\prime}$ is not contained in $\tilde{\beta}\left(\rho_{1}\right) \subset \tilde{Z}_{0}$.

Now we claim that $\rho_{1}$ is trivial. Otherwise, if $\rho_{1}$ is nontrivial, namely, $\tilde{e} \subset \tilde{Z}_{0}$ and $\tilde{e} \neq \tilde{e}^{\prime}$. Write $\rho^{\prime}=\rho_{1} \rho_{2}$ for $\rho_{2}=\rho^{\prime} \backslash \rho_{1}$ the sub-ray of $\rho^{\prime}$ with origin $\rho_{2}(0)=\tilde{e}^{\prime}(0)$. Since $\tilde{v}^{\prime}$ is the only fixed point in $\rho^{\prime}$ and $\tilde{\beta}\left(\rho_{1}\right)$ does not contain the edge $\tilde{e}^{\prime}$, we have $\tilde{\beta}\left(\rho_{2}\right)$ contains $\rho_{2}$ as a proper sub-ray, that is, $\tilde{\beta}\left(\tilde{e}^{\prime}(0)\right)$ is not in the same connected component of $\tilde{Z} \backslash \tilde{e}^{\prime}(0)$ as $\tilde{e}^{\prime}(1)$. Then $\tilde{e}^{\prime}(0)$ and any point $y \in \rho^{\prime}$ sufficiently close to $\partial \tilde{Z}$ satisfy the hypothesis of the fixed point criterion, and $\tilde{\beta}$ has a fixed point in the path $\left[\tilde{e}^{\prime}(0), y\right] \subset \rho^{\prime}$. It contradicts that $\tilde{v}^{\prime}$ is the unique fixed point in $\rho^{\prime}$.

Therefore, $\rho_{1}$ is trivial, that is, $\tilde{e}=\tilde{e}^{\prime} \subset \tilde{Z} \backslash \tilde{Z}_{0}$, then $\rho^{\prime}=\tilde{\beta}^{\infty}(\tilde{e})=\bar{j}\left(W_{\tilde{e}}\right)$. It follows $\mathscr{W}=\mathscr{W}_{e}$ for $e=q(\tilde{e}) \in \Delta(\mathbf{F})$, so claim (3) holds.

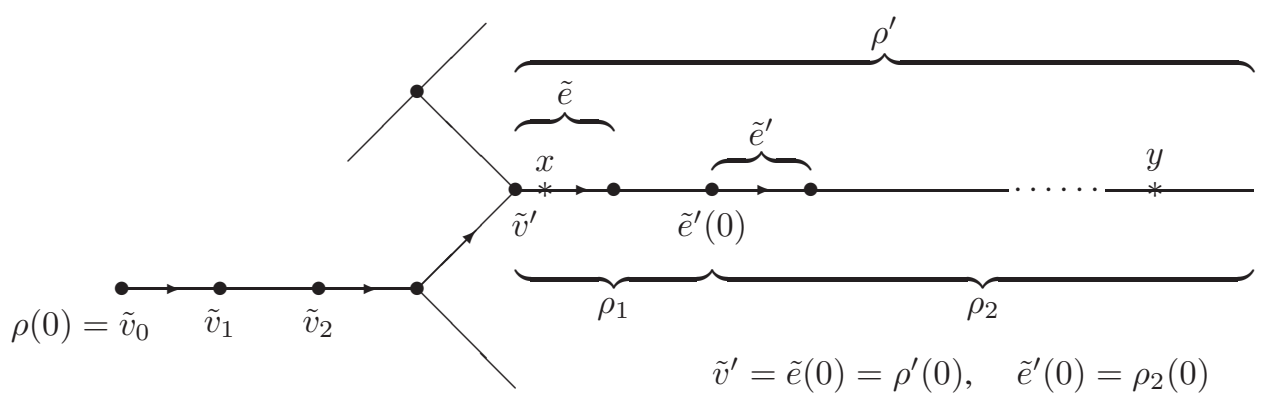

FIGURE 2. The ray $\rho$ and its sub-ray $\rho^{\prime}$

4.4. RTT map of Type 1 and Type 2. Let $\beta:\left(Z, Z_{0}\right) \rightarrow\left(Z, Z_{0}\right)$ be an RTT map, and $\beta_{0}:=\left.\beta\right|_{Z_{0}}$. If $\beta$ is of Type 1 , then $\left.\beta_{\pi}\right|_{\pi_{1}\left(Z_{0}, v\right)}=\left(\beta_{0}\right)_{\pi}: \pi_{1}\left(Z_{0}, v\right) \rightarrow \pi_{1}\left(Z_{0}, v\right)$ and $\beta_{\pi}\left(\pi_{1}(Z, v)\right) \subset \pi_{1}\left(Z_{0}, v\right)$. So $\operatorname{Fix}\left(\beta_{\pi}\right)=\operatorname{Fix}\left(\beta_{0}\right)_{\pi}$ and $a\left(\beta_{\pi}\right)=a\left(\beta_{0}\right)_{\pi}$ immediately.

For an RTT map of Type 2, we have

Lemma 4.4. Let $\beta:\left(Z, Z_{0}\right) \rightarrow\left(Z, Z_{0}\right)$ be an RTT map of Type 2 . Then every attracting fixed word of $\beta_{\pi}$ is equivalent to a one of $\left(\beta_{0}\right)_{\pi}$.

Proof. Pick a lifting $\tilde{\beta}: \tilde{Z} \rightarrow \tilde{Z}$ as in Section 4.3 
For an attracting fixed word $W$ of $\beta_{\pi}$, by the above Assertion $\star$, there exists an attracting fixed point $\bar{j}(W) \in \partial \tilde{Z}$ of $\tilde{\beta}$. Suppose $\rho:[0,+\infty] \rightarrow \tilde{Z}$ is a ray representing $\bar{j}(W)$ with origin $\rho(0)=\tilde{v}_{0}$. Since $\bar{j}(W)$ is attracting (under the metric $d$ on $\tilde{Z}$ that every edge has length 1), as in the proof of claim (3) in Lemma4.3, we can show the set $S_{\rho}:=\rho \cap \operatorname{Fix} \tilde{\beta}$ is finite, and let $\rho^{\prime}$ be the sub-ray of $\rho$ such that the origin $\rho^{\prime}(0)=\tilde{v}^{\prime}$ is the only fixed point in $\rho^{\prime}$.

Let $\tilde{e}$ be the initial oriented edge of $\rho^{\prime}$ with $\tilde{e}(0)=\tilde{v}^{\prime}$, as in the proof of claim (3) in Lemma 4.3 we also have $D \tilde{\beta}(\tilde{e})=\tilde{e}$ and $\left[\tilde{\beta}\left(\rho^{\prime}\right)\right]=\rho^{\prime}$. Recall that $\beta$ is an RTT of Type 2, if $\tilde{e} \subset \tilde{Z} \backslash \tilde{Z}_{0}$, then $Z \backslash Z_{0}$ has only one edge $q(\tilde{e})$, and hence $\tilde{\beta}(\tilde{e})=\tilde{e}$, contradicting that the origin $\tilde{v}^{\prime}$ is the only fixed point in $\rho^{\prime}$. Therefore, $\tilde{e} \subset \tilde{Z}_{0}$.

If $\rho^{\prime} \not \subset \tilde{Z}_{0}$, it contains an oriented edge $\tilde{e}^{\prime} \subset \tilde{Z} \backslash \tilde{Z}_{0}$. Since $\tilde{\beta}\left(\tilde{e}^{\prime}\right) \subset \tilde{Z} \backslash \tilde{Z}_{0}$ and $\tilde{\beta}\left(\tilde{Z}_{0}\right) \subset$ $\tilde{Z}_{0}$, by the same argument as in the proof of claim (3) in Lemma 4.3, we can show $\tilde{\beta}$ has another fixed point but not $\tilde{v}^{\prime}$ in $\rho^{\prime}$, contradicting that $\tilde{v}^{\prime}$ is the only fixed point in $\rho^{\prime}$. Therefore, the sub-ray $\rho^{\prime} \subset \tilde{Z}_{0}$, and represents an attracting fixed point $\bar{j}\left(W^{\prime}\right)$ of $\tilde{\beta}_{0}$ for $W^{\prime}$ an attracting fixed word of $\left(\tilde{\beta}_{0}\right)_{\pi}$. It follows that the attracting fixed point $W$ of $\tilde{\beta}$ is equivalent to $W^{\prime}$. So Lemma4.4 is true.

4.5. Property of $\operatorname{ichr}(\mathbf{F})$ of RTT map. In this subsection, we give some relations between $\operatorname{ichr}\left(\beta_{0}, \mathbf{F}_{0}\right)$ and $\operatorname{ichr}(\beta, \mathbf{F})$.

Proposition 4.5. Let $\beta:\left(Z, Z_{0}\right) \rightarrow\left(Z, Z_{0}\right)$ be an RTT map as in Thereom 4.1] and $\beta_{0}:=\left.\beta\right|_{Z_{0}}$. If there exists an indivisible $\beta$-Nielsen path $p$ that intersects $Z \backslash Z_{0}$, it is unique. (In Type 2 when $Z \backslash Z_{0}$ is a single edge e, take $p=e$, and consider that e gets initially expanded along itself on one tip and shrinks on the other.) There are three possible cases.

(i) No such path p exists (as always in Type 1, in Type 2 when $Z \backslash Z_{0}$ has more than one edge, and possibly in Type 3). Then the $\beta$-fixed point classes are the same as the $\beta_{0}$-fixed point classes, and

$$
\operatorname{rk}\left(\beta, \mathbf{F}_{0}\right)=\operatorname{rk}\left(\beta_{0}, \mathbf{F}_{0}\right), \quad a\left(\beta, \mathbf{F}_{0}\right)=a\left(\beta_{0}, \mathbf{F}_{0}\right)+\delta\left(\mathbf{F}_{0}\right)
$$

for all $\beta_{0}$-fixed point classes $\mathbf{F}_{0}$.

(ii) The path $p$ connects two different $\beta_{0}$-fixed point classes $\mathbf{F}_{1}^{\prime}$ and $\mathbf{F}_{2}^{\prime}$. Then the $\beta$-fixed point classes are the same as the $\beta_{0}$-fixed point classes, except that $\mathbf{F}_{1}^{\prime}$ and $\mathbf{F}_{2}^{\prime}$ combine into a single $\beta$-fixed point class $\mathbf{F}^{\prime}=\mathbf{F}_{1}^{\prime} \cup \mathbf{F}_{2}^{\prime}$. We have

$$
\operatorname{rk}\left(\beta, \mathbf{F}^{\prime}\right)=\operatorname{rk}\left(\beta_{0}, \mathbf{F}_{1}^{\prime}\right)+\operatorname{rk}\left(\beta_{0}, \mathbf{F}_{2}^{\prime}\right), a\left(\beta, \mathbf{F}^{\prime}\right)=a\left(\beta_{0}, \mathbf{F}_{1}^{\prime}\right)+a\left(\beta_{0}, \mathbf{F}_{2}^{\prime}\right)+\delta\left(\mathbf{F}^{\prime}\right)-1,
$$

and for each $\beta$-fixed point class $\mathbf{F}_{0} \neq \mathbf{F}^{\prime}$,

$$
\operatorname{rk}\left(\beta, \mathbf{F}_{0}\right)=\operatorname{rk}\left(\beta_{0}, \mathbf{F}_{0}\right), \quad a\left(\beta, \mathbf{F}_{0}\right)=a\left(\beta_{0}, \mathbf{F}_{0}\right)+\delta\left(\mathbf{F}_{0}\right) .
$$

(iii) The path $p$ has both endpoints in a $\beta_{0}$-fixed point class $\mathbf{F}_{0}^{\prime}$. Then the $\beta$-fixed point classes are the same as the $\beta_{0}$-fixed point classes, and

$$
\operatorname{rk}\left(\beta, \mathbf{F}_{0}^{\prime}\right)=\operatorname{rk}\left(\beta_{0}, \mathbf{F}_{0}^{\prime}\right)+1, \quad a\left(\beta, \mathbf{F}_{0}^{\prime}\right)=a\left(\beta_{0}, \mathbf{F}_{0}^{\prime}\right)+\delta\left(\mathbf{F}_{0}^{\prime}\right)-1,
$$


and for each $\beta$-fixed point class $\mathbf{F}_{0} \neq \mathbf{F}_{0}^{\prime}$,

$$
\operatorname{rk}\left(\beta, \mathbf{F}_{0}\right)=\operatorname{rk}\left(\beta_{0}, \mathbf{F}_{0}\right), \quad a\left(\beta, \mathbf{F}_{0}\right)=a\left(\beta_{0}, \mathbf{F}_{0}\right)+\delta\left(\mathbf{F}_{0}\right) .
$$

Proof. The proof of the equalities of $a(\cdot)$ relies mainly on Lemma 4.3 and Lemma 4.4 Although the equalities of $\operatorname{rk}(\cdot)$ have been proved in [JWZ, Corollary BH], to be referenced easily, we state them in the following.

At first, let's review some notations.

For a fixed point $v$ of $\beta$, as in Definition 3.4, let $\beta_{v}: \pi_{1}(Z, v) \rightarrow \pi_{1}(Z, v)$ be the induced natural injective endomorphism and $\left(\beta_{0}\right)_{v}=\left.\beta_{v}\right|_{\pi_{1}\left(Z_{0}, v\right)}: \pi_{1}\left(Z_{0}, v\right) \rightarrow \pi_{1}\left(Z_{0}, v\right)$. For $\phi \in\left\{\beta_{v},\left(\beta_{0}\right)_{v}\right\}$, let $\mathcal{A}(\phi)$ be the set of attracting fixed words of $\phi$. The set $\mathscr{A}(\phi)=$ $\mathcal{A}(\phi) / \sim$ is the equivalence classes of attracting fixed words of $\phi$ (where $W, V \in \mathcal{A}(\phi)$ are equivalent if and only if $W=\omega V$ for some $\omega \in \operatorname{Fix}(\phi))$. Clearly, the inclusion $\iota$ : $\left(Z_{0}, v\right) \hookrightarrow(Z, v)$ is an inj-morphism from $\beta_{0}:\left(Z_{0}, v\right) \rightarrow\left(Z_{0}, v\right)$ to $\beta:(Z, v) \rightarrow(Z, v)$, then by Fact 2 in Section 3.4 Fix $\left(\beta_{0}\right)_{v} \subset \operatorname{Fix}\left(\beta_{v}\right)$ and $\mathcal{A}\left(\beta_{0}\right)_{v} \subset \mathcal{A}\left(\beta_{v}\right)$.

Moreover, for two attracting fixed words $W, V \in \mathcal{A}\left(\beta_{0}\right)_{v}$ that are in the same equivalence class in $\mathscr{A}\left(\beta_{v}\right)$, i.e., $W=\omega V$ for some $\omega \in \operatorname{Fix}\left(\beta_{v}\right)$, since $W, V \in \partial \pi_{1}\left(Z_{0}, v\right)$ and $\pi_{1}(Z, v)=\pi_{1}\left(Z_{0}, v\right) * G$ for $G$ a free subgroup of $\pi_{1}(Z, v)$, we have $\omega \in \operatorname{Fix}\left(\beta_{v}\right) \cap$ $\pi_{1}\left(Z_{0}, v\right)=\operatorname{Fix}\left(\beta_{0}\right)_{v}$. So $W, V$ are also in the same equivalence class in $\mathscr{A}\left(\beta_{0}\right)_{v}$. Therefore, the inclusion $\mathcal{A}\left(\beta_{0}\right)_{v} \subset \mathcal{A}\left(\beta_{v}\right)$ induces an injection

$$
\bar{\iota}_{v}: \mathscr{A}\left(\beta_{0}\right)_{v} \hookrightarrow \mathscr{A}\left(\beta_{v}\right) .
$$

Recall that $a(\beta, \mathbf{F})=a\left(\beta_{v}\right)=\# \mathscr{A}\left(\beta_{v}\right)$ for $v \in \mathbf{F}$.

Case (i) Since every Nielsen path is a product of indivisible Nielsen paths, it lies in $Z_{0}$. So for any $v \in \mathbf{F}_{0}$, $\operatorname{Fix}\left(\beta_{v}\right)=\operatorname{Fix}\left(\beta_{0}\right)_{v}$ and $\operatorname{rk}\left(\beta, \mathbf{F}_{0}\right)=\operatorname{rk}\left(\beta_{0}, \mathbf{F}_{0}\right)$.

If $\beta$ is of Type 1 or Type 2 , then $\delta\left(\mathbf{F}_{0}\right)=0$ and $\mathscr{A}\left(\beta_{v}\right)=\mathscr{A}\left(\beta_{0}\right)_{v}$ by Lemma 4.4 if $\beta$ is of Type 3, then by Lemma 4.3, the map $e \mapsto \mathscr{W}_{e}$ defines a bijection from $\Delta\left(\mathbf{F}_{0}\right)$ to $\mathscr{A}\left(\beta_{v}\right) \backslash \mathscr{A}\left(\beta_{0}\right)_{v}$. In either case, we would have $a\left(\beta, \mathbf{F}_{0}\right)=a\left(\beta_{0}, \mathbf{F}_{0}\right)+\delta\left(\mathbf{F}_{0}\right)$.

Case (ii) For the equalities of $\mathbf{F}_{0}$, the situation is the same as the one in Case (i). Now we consider $\mathbf{F}^{\prime}=\mathbf{F}_{1}^{\prime} \cup \mathbf{F}_{2}^{\prime}$. Suppose the path $p$ goes from $a \in \mathbf{F}_{1}^{\prime}$ to $b \in \mathbf{F}_{2}^{\prime}$.

For the $\operatorname{rk}\left(\beta, \mathbf{F}^{\prime}\right)$ equation it suffices to show that the natural homomorphism

$$
\eta: \operatorname{Fix}\left(\beta_{0}\right)_{a} * \operatorname{Fix}\left(\beta_{0}\right)_{b} \rightarrow \operatorname{Fix}\left(\beta_{a}\right), \quad[u] \mapsto[u],[v] \mapsto p_{\#}[v]:=[p v \bar{p}],
$$

is an isomorphism, where $u$ and $v$ are Nielsen paths in $Z_{0}$ at $a$ and $b$, respectively, and [.] denotes loop class. First observe that $\eta$ is injective. In fact, the $\eta$-image of any nontrivial element of the left hand side is represented by a product $w=u_{1} p v_{1} \bar{p} \cdots u_{l} p v_{l} \bar{p} u_{l+1}$, where $u_{i}$ and $v_{i}$ are Nielsen paths in $Z_{0}$ at $a$ and $b$, respectively. (We allow that $u_{1}$ and $u_{l+1}$ be trivial, but assume the others are nontrivial.) By Property of Type 2 or 3(c) in Theorem 4.1 both tips of $\mathrm{p}$ are in $Z \backslash Z_{0}$, so $w$ is an immersed Nielsen path and represents a nontrivial element in the left hand side. 
On the other hand, any nontrivial element of the right hand side is represented by a concatenation of $\beta$-Nielsen paths, hence by a product like the $w$ above, so $\eta$ is surjective. Thus the desired isomorphism is established.

Below we prove the equalities of $a\left(\beta, \mathbf{F}^{\prime}\right)$.

By the bijective correspondence Assertion $\star$, two attracting fixed words $W \in \mathcal{A}\left(\beta_{0}\right)_{a}$ and $W^{\prime} \in \mathcal{A}\left(\beta_{0}\right)_{b}$ can be represented by two rays $\rho, \rho^{\prime} \subset \tilde{Z}_{0}:=q^{-1}\left(Z_{0}\right) \subset \tilde{Z}$ with origin $\tilde{a}, \tilde{b} \in \operatorname{Fix} \tilde{\beta}$, respectively. So the inclusions induce two injections

$$
\begin{gathered}
\bar{\iota}_{a}: \mathscr{A}\left(\beta_{0}\right)_{a} \hookrightarrow \mathscr{A}\left(\beta_{a}\right), \mathscr{W}_{\rho} \mapsto \mathscr{W}_{\rho}, \\
\bar{\iota}_{b}: \mathscr{A}\left(\beta_{0}\right)_{b} \hookrightarrow \mathscr{A}\left(\beta_{a}\right), \mathscr{W}_{\rho^{\prime}} \mapsto \mathscr{W}_{\tilde{p} \rho^{\prime}},
\end{gathered}
$$

where $\mathscr{W}_{\rho}$ denotes the equivalence class of the attracting fixed word represented by $\rho$, and $\bar{\iota}_{b}$ can be thought as a composition $\mathscr{A}\left(\beta_{0}\right)_{b} \hookrightarrow \mathscr{A}\left(\beta_{b}\right) \longleftrightarrow \mathscr{A}\left(\beta_{a}\right)$, which is well-defined because the lifiting $\tilde{p}$ of $p$ is a $\tilde{\beta}$-Nielsen path from $\tilde{a}$ to $\tilde{b}$ and hence $\beta\left(\tilde{p} \rho^{\prime}\right)=\tilde{p} \rho^{\prime}$ represents an attracting fixed word in $\mathcal{A}(\beta)_{a}$.

Furthermore, we claim $\bar{\iota}_{a}\left(\mathscr{A}\left(\beta_{0}\right)_{a}\right) \cap \bar{\iota}_{b}\left(\mathscr{A}\left(\beta_{0}\right)_{b}\right)=\emptyset$. It implies an injection

$$
\bar{\iota}:=\bar{\iota}_{a} \sqcup \bar{\iota}_{b}: \mathscr{A}\left(\beta_{0}\right)_{a} \sqcup \mathscr{A}\left(\beta_{0}\right)_{b} \hookrightarrow \mathscr{A}\left(\beta_{a}\right) .
$$

Otherwise, there exist rays $\rho, \rho^{\prime} \subset \tilde{Z}_{0}$ with $\tilde{\beta}$-fixed origins $\tilde{a} \in q^{-1}(a)$ and $\tilde{b} \in q^{-1}(b)$ respectively, such that $\rho$ and $\tilde{p} \rho^{\prime}$ represent two equivalent attracting fixed words in $\mathcal{A}\left(\beta_{a}\right)$, that is, there exist an word $w \in \operatorname{Fix}\left(\beta_{a}\right)$, such that the two rays $\rho$ and $w\left(\tilde{p} \rho^{\prime}\right)$ represent the same end in $\partial \tilde{Z}$. It implies that $\gamma:=\rho \cap w\left(\tilde{p} \rho^{\prime}\right) \subset \tilde{Z}_{0}$ is a ray. Note that $w\left(\tilde{p} \rho^{\prime}\right)=w(\tilde{p}) \cdot w\left(\rho^{\prime}\right)$ and $\tilde{p}$ is a lifting of the indivisible Nielsen path $p$, then the two tips of $w(\tilde{p}) \subset \tilde{Z} \backslash \tilde{Z}_{0}$ and $\gamma \subset w\left(\rho^{\prime}\right) \subset \tilde{Z}_{0}$. Let $\rho_{1}:=\rho \backslash \gamma$ and $\rho_{1}^{\prime}:=w\left(\rho^{\prime}\right) \backslash \gamma$, then $\rho_{1} \bar{\rho}_{1}^{\prime} \subset \tilde{Z}_{0}$ is a path from $\tilde{a}$ to $w(\tilde{b})$. Recall $w \in \operatorname{Fix}\left(\beta_{a}\right)$ and $\tilde{b} \in \operatorname{Fix} \tilde{\beta}$, then $\tilde{\beta}(w(\tilde{b}))=\beta_{a}(w)(\tilde{\beta}(\tilde{b}))=w(\tilde{b})$ is a fixed point of $\tilde{\beta}$. It implies that $\rho_{1} \bar{\rho}_{1}^{\prime} \subset \tilde{Z}_{0}$ is a Nielsen path from $\tilde{a}$ to $w(\tilde{b})$, contradicting that $a \in \mathbf{F}_{1}^{\prime}$ and $b \in \mathbf{F}_{2}^{\prime}$ belong to distinct fixed point classes of $\beta_{0}$. So the claim is true.

Using the injection $\bar{\iota}$ in equation (4.4), we can compute $a\left(\beta, \mathbf{F}^{\prime}\right)$ immediately. There are two cases:

(a) $p$ is the unique edge $e$ of $Z \backslash Z_{0}$ in Type 2 (recall that in this case $\delta\left(\mathbf{F}^{\prime}\right)=1$ because $e$ gets initially expanded along itself on only one tip). By Lemma $4.4 \bar{\imath}$ is a bijection. So

$$
a\left(\beta, \mathbf{F}^{\prime}\right)=a\left(\beta_{0}, \mathbf{F}_{1}^{\prime}\right)+a\left(\beta_{0}, \mathbf{F}_{2}^{\prime}\right)=a\left(\beta_{0}, \mathbf{F}_{1}^{\prime}\right)+a\left(\beta_{0}, \mathbf{F}_{2}^{\prime}\right)+\delta\left(\mathbf{F}^{\prime}\right)-1 .
$$

(b) $p$ is the unique indivisible Nielsen path in Type 3. Follows from Lemma4.3, each element of $\mathscr{A}\left(\beta_{a}\right) \backslash \bar{l}\left(\mathscr{A}\left(\beta_{0}\right)_{a} \sqcup \mathscr{A}\left(\beta_{0}\right)_{b}\right)$ can be represented by $\mathscr{W}_{e}$ for a unique edge $e \in \Delta\left(\mathbf{F}^{\prime}\right)$, except that $e_{1}, e_{2} \in \Delta\left(\mathbf{F}^{\prime}\right)$ are the two tips of the indivisible Nielsen path $p$ and they give the same element $\mathscr{W}_{e_{1}}=\mathscr{W}_{e_{2}}$, and conversely. Therefore, we also have

$$
a\left(\beta, \mathbf{F}^{\prime}\right)=a\left(\beta_{0}, \mathbf{F}_{1}^{\prime}\right)+a\left(\beta_{0}, \mathbf{F}_{2}^{\prime}\right)+\delta\left(\mathbf{F}^{\prime}\right)-1 .
$$


Case (iii) For the equalities of $\mathbf{F} \neq \mathbf{F}_{0}^{\prime}$, the situation is the same as the one in Case (i).

Suppose the path $p$ goes from $a$ to $b$ both in $\mathbf{F}_{0}^{\prime}$. By the similar argument as in case (ii), we can show $\eta$

$$
\eta: \operatorname{Fix}\left(\beta_{0}\right)_{a} * J \rightarrow \operatorname{Fix}\left(\beta_{a}\right), \text { defined by }[u] \mapsto[u],[t] \mapsto[t]
$$

is an isomorphism, where the loop class $[u] \in \operatorname{Fix}\left(\beta_{0}\right)_{a}$ and $J$ is the infinite cyclic group generated by the loop class $[t]$ represented by the loop $t:=p q$ when $a \neq b$, but taking $t:=p$ when $a=b$. It implies $\operatorname{rk}\left(\beta, \mathbf{F}_{0}^{\prime}\right)=\operatorname{rk}\left(\beta_{0}, \mathbf{F}_{0}^{\prime}\right)+1$.

By equation (4.3), $\bar{\iota}_{a}: \mathscr{A}\left(\beta_{0}\right)_{a} \hookrightarrow \mathscr{A}\left(\beta_{a}\right)$ is an injection. Then by the similar argument as the one in the above Case (a) and Case (b), we have $a\left(\beta, \mathbf{F}_{0}^{\prime}\right)=a\left(\beta_{0}, \mathbf{F}_{0}^{\prime}\right)+\delta\left(\mathbf{F}_{0}^{\prime}\right)-$ 1.

As a corollary of the above Proposition 4.5 , we have

Corollary 4.6. Let $\beta:\left(Z, Z_{0}\right) \rightarrow\left(Z, Z_{0}\right)$ be an RTT map as in Theorem 4.1 and $\beta_{0}:=$ $\left.\beta\right|_{Z_{0}}: Z_{0} \rightarrow Z_{0}$. Then for every nonempty fixed point class $\mathbf{F}$ of $\beta$, we have

$$
\operatorname{ichr}(\beta, \mathbf{F})=\operatorname{ichr}\left(\beta_{0}, \mathbf{F}\right)-\delta(\mathbf{F}),
$$

where $\operatorname{ichr}\left(\beta_{0}, \mathbf{F}\right):=\sum_{i=1}^{k} \operatorname{ichr}\left(\beta_{0}, \mathbf{F}_{i}\right)$ if $\mathbf{F}=\sqcup \mathbf{F}_{i}$ is a union of $k \leq 2 \beta_{0}$-fixed point classes $\mathbf{F}_{i}, i=1, \ldots, k$.

Proof. Since $\operatorname{ichr}(\mathbf{F})=1-\operatorname{rk}(\mathbf{F})-a(\mathbf{F})$, the conclusion can be checked immediately case by case in Proposition 4.5

\section{Proofs of THEOREMS}

Now we prove main theorems of this paper.

Proof of Theorem 1.2 Let $f$ be a $\pi_{1}$-injective selfmap of a connected finite graph $X$, and $\mathbf{F}$ a fixed point class of $f$. Since ind $(\mathbf{F})$ and ichr $(\mathbf{F})$ both have homotopy and commutation invariance (see Section 3.4), it suffices to prove Theorem 1.2 for an RTT map $\beta:\left(Z, Z_{0}\right) \rightarrow\left(Z, Z_{0}\right)$ in Theorem 4.1 where $Z$ is a connected finite graph without vertices of valence 1 and with $\chi(Z)=\chi(X)$. Assume $\chi(Z) \leq 0$ because it is trivial for $\chi(Z)>0$.

If $\chi(Z)=0$, i.e., $Z$ is a circle, then by Example 3.8, we have $\operatorname{ind}(\mathbf{F})=\operatorname{ichr}(\mathbf{F})$ for every fixed point class $\mathbf{F}$ of $\beta$. So Theorem 1.2 holds.

If $\chi(Z)<0$ and $\mathbf{F}$ is empty, then $\operatorname{ind}(\mathbf{F})=0$ while $0 \leq \operatorname{ichr}(\mathbf{F}) \leq 1$ by Proposition 3.3 and hence Theorem 1.2 holds. Now we assume $\mathbf{F}$ is a nonempty fixed point class of $\beta$ in the following. Let $Z_{1}, \ldots, Z_{n}$ be the connected components of $Z_{0}$. Suppose the $\beta$ invariant ones are $Z_{1}, \ldots, Z_{k}$. Denote $\beta_{0}:=\left.\beta\right|_{Z_{0}}: Z_{0} \rightarrow Z_{0}$ and $\beta_{i}:=\left.\beta\right|_{Z_{i}}: Z_{i} \rightarrow Z_{i}$ for $i=1, \ldots, k$. Since $Z$ is a connected graph without vertices of valence 1 , and $Z_{0}$ is a 
proper subgraph containing all the vertices of $Z$, it is easy to see that all $\chi\left(Z_{i}\right)>\chi(Z)$. So, working inductively, we may assume that Theorem 1.2 is true for every $\beta_{i}$, that is,

$$
\operatorname{ind}\left(\beta_{i}, \mathbf{F}_{i}\right) \leq \operatorname{ichr}\left(\beta_{i}, \mathbf{F}_{i}\right)
$$

for every fixed point class $\mathbf{F}_{i}$ of $\beta_{i}$.

For the nonempty fixed point class $\mathbf{F}$ of $\beta$, note that $\mathbf{F}$ is a union of finitely many (in fact at most 2 by Proposition 4.5 $\beta_{0}$-fixed point classes $\mathbf{F}_{i}$, each is contained in a unique component $Z_{i}$ and is also a fixed point class of $\beta_{i}$, so $\operatorname{ind}\left(\beta_{0}, \mathbf{F}_{i}\right)=\operatorname{ind}\left(\beta_{i}, \mathbf{F}_{i}\right)$ and $\operatorname{ichr}\left(\beta_{i}, \mathbf{F}_{i}\right)=\operatorname{ichr}\left(\beta_{0}, \mathbf{F}_{i}\right)$. Therefore, by the inductive hypothesis (equation 5.1), we have

$$
\operatorname{ind}\left(\beta_{0}, \mathbf{F}_{i}\right) \leq \operatorname{ichr}\left(\beta_{0}, \mathbf{F}_{i}\right) .
$$

Combine the above inequality, equation 4.1) with Corollary 4.6, Theorem 1.2 holds.

Proof of Theorem 1.3 Since ind $(\mathbf{F})$ and ichr $(\mathbf{F})$ both have homotopy invariance, it suffices to assume that $f$ is an RTT map $\beta:\left(Z, Z_{0}\right) \rightarrow\left(Z, Z_{0}\right)$ in Theorem 4.1 where $\chi(Z)=\chi(X)=-1$.

If $\mathbf{F}$ is inessential, then the conclusion is clear by Theorem 1.2 .

Now we assume that $\mathbf{F}$ is essential, then it is a nonempty fixed point class of $\beta$. Using the same notations $Z_{1}, \ldots, Z_{n}$ and $\beta_{0}, \beta_{1}, \ldots, \beta_{k}$ as in the above proof of Theorem 1.2 we have $1 \geq \chi\left(Z_{i}\right)>\chi(Z)=-1$, and $\mathbf{F}$ is a finite union of $\beta_{0}$-fixed point classes $\mathbf{F}_{i}$ which is contained in a unique component $Z_{i}$ and is also a fixed point class of $\beta_{i}$.

If $\chi\left(Z_{i}\right)=1$, then $Z_{i}$ is a vertex of $Z$, and hence every fixed point class $\mathbf{F}_{i}$ of $\beta_{i}$ : $\mathbb{Z}_{i} \rightarrow Z_{i}$ consists of a single point with $\operatorname{ind}\left(\beta_{i}, \mathbf{F}_{i}\right)=\operatorname{ichr}\left(\beta_{i}, \mathbf{F}_{i}\right)=1$. If $\chi\left(Z_{i}\right)=0$, after a homotopy, $Z_{i}$ is a circle. From Example 3.8 we have $\operatorname{ind}\left(\beta_{i}, \mathbf{F}_{i}\right)=\operatorname{ichr}\left(\beta_{i}, \mathbf{F}_{i}\right)$ for every fixed point class $\mathbf{F}_{i}$ of $\beta_{i}$. Therefore, we always have

$$
\operatorname{ind}\left(\beta_{0}, \mathbf{F}_{i}\right)=\operatorname{ind}\left(\beta_{i}, \mathbf{F}_{i}\right)=\operatorname{ichr}\left(\beta_{i}, \mathbf{F}_{i}\right)=\operatorname{ichr}\left(\beta_{0}, \mathbf{F}_{i}\right) .
$$

Combine the above equality, equation (4.1) with Corollary 4.6 the conclusion ind $(\mathbf{F})=$ $\operatorname{ichr}(\mathbf{F})$ holds.

Remark 5.1. From the above two proofs, it is clear that Conjecture 1.4 is true if and only if $\operatorname{ind}(\mathbf{F})=\operatorname{ichr}(\mathbf{F})=0$ holds for every empty fixed point class $\mathbf{F}$.

Proof of Theorem 2.6. For an injective endomorphism $\phi$ of $F_{n}$, there exists a $\pi_{1}$-injective selfmap $f:(X, *) \rightarrow(X, *)$ of a connected finite graph $X$ with fundamental group $\pi_{1}(X, *)=F_{n}$ such that $\phi=f_{*}: \pi_{1}(X, *) \rightarrow \pi_{1}(X, *),[c] \mapsto[f \circ c]$. Moreover, for any $i_{[w]} \circ \phi \in \operatorname{Inn} \phi$, we have

$$
i_{[w]} \circ \phi=f_{w}: \pi_{1}(X, *) \rightarrow \pi_{1}(X, *), \quad[c] \mapsto[w(f \circ c) \bar{w}],
$$

where $w$ is a loop based at $*$ and the loop class $[w] \in \pi_{1}(X, *)$.

Two endomorphisms $\psi^{\prime}=i_{\left[w^{\prime}\right]} \circ \phi$ and $\psi=i_{[w]} \circ \phi$ in Inn $\phi$ are similar (see Section 2.2 for a definition) if and only if there exists $[u] \in \pi_{1}(X, *)$ for $u$ a loop based at $*$ such 
that $\psi^{\prime}=i_{[u]} \circ \psi \circ\left(i_{[u]}\right)^{-1}$, that is,

$$
i_{\left[w^{\prime}\right]} \circ \phi=i_{[u]} \circ i_{[w]} \circ \phi \circ i_{[\bar{u}]}=i_{[u w(f \circ \bar{u})]} \circ \phi .
$$

Since $\phi\left(F_{n}\right) \cong F_{n}$ and the centralizer of any nontrivial element in a free group $F_{n}$ of rank $n \geq 2$ is free cyclic, the above Equation (5.2) holds if and only if $\left[w^{\prime}\right]=[u w(f \circ \bar{u})]$, that is, the $f$-routes $w^{\prime}$ and $w$ are associated with the same fixed point class $\mathbf{F}$ (see Definition 3.5). Therefore, we have a bijection $\mathbf{F} \mapsto[\psi]$ between the fixed point classes of $f$ and the similarity classes contained in $\operatorname{Inn} \phi$, with

$$
\operatorname{ichr}(\mathbf{F})=1-\operatorname{rk}(\mathbf{F})-a(\mathbf{F})=1-\operatorname{rkFix}(\psi)-a(\psi) .
$$

By Corollary 1.6 , we have

$$
\sum_{[\psi] \subset \operatorname{Inn} \phi} \max \{0, \operatorname{rkFix}(\psi)+a(\psi) / 2-1\} \leq n-1 .
$$

So Theorem 2.6 holds.

Proof of Theorem 1.7 The conclusion follows from Example 2.7 immediately if the rank $n=1$. Now we assume $n \geq 2$. As in the above proof of Theorem 2.6 there exists a $\pi_{1}$-injective selfmap $f: X \rightarrow X$ of a connected finite graph $X$ with fundamental group $\pi_{1}(X)=F_{n}$ such that $\phi=f_{*}: \pi_{1}(X, *) \rightarrow \pi_{1}(X, *)$, and the abelianization of $\phi$,

$$
\phi^{\mathrm{ab}}=f_{\sharp}: H_{1}(X) \rightarrow H_{1}(X)=\mathbb{Z}^{n},
$$

the endomorphism induced by $f$ on the homology group $H_{1}(X)$. By the famous LefschetzHopf fixed point theorem, the Lefschetz number

$$
L(f)=1-\operatorname{tr}\left(\phi^{\mathrm{ab}}\right)=\sum_{\mathbf{F} \in \operatorname{Fpc}(f)} \operatorname{ind}(f, \mathbf{F}) .
$$

If $\operatorname{tr}\left(\phi^{\mathrm{ab}}\right)<1$, then there exists $\mathbf{F} \in \operatorname{Fpc}(f)$ such that $\operatorname{ind}(f, \mathbf{F})>0$, and hence $\operatorname{ichr}(f, \mathbf{F})>0$ by Theorem 1.2 So by Equation [5.3), there exists $c \in F_{n}$ such that $\operatorname{rkFix}\left(i_{c} \circ \phi\right)=a\left(i_{c} \circ \phi\right)=0$.

If $n=2$ and $\operatorname{tr}\left(\phi^{\mathrm{ab}}\right)>1$, then there exists $\mathbf{F} \in \operatorname{Fpc}(f)$ with $\operatorname{ind}(f, \mathbf{F})<0$, and hence $\operatorname{ichr}(f, \mathbf{F})<0$ by Theorem 1.3. So by Equation [5.3, there exists $c \in F_{n}$ such that $\operatorname{rkFix}\left(i_{c} \circ \phi\right)+a\left(i_{c} \circ \phi\right)>1$.

Proof of Theorem 1.8 The conclusion follows from Example 2.7 immediately if the rank $n=1$. For $n \geq 2$, pick one of the summands in the inequality of Theorem 2.6 we have that $\max \{0, \operatorname{rkFix}(\phi)+a(\phi) / 2-1\} \leq n-1$. This is what we need to show.

\section{EXAMPLES}

The following example shows that the bounds in Theorem 1.8 and Theorem 2.6 are sharp. 
Example 6.1. Let $f:\left(R_{n}, *\right) \rightarrow\left(R_{n}, *\right)$ be a $\pi_{1}$-injective selfmap of the graph $R_{n}$ with one vertex $*$ and $n \geq 1$ edges $a_{1}, \ldots, a_{n}$, such that $f\left(a_{i}\right)=a_{i}^{2}$ for $i=1, \ldots, n$.

Note that $f$ is an RTT map with $Z=R_{n}$ and $Z_{0}=*$. Then $*$ is the unique nonempty fixed point class of $f$, and $\Delta(*)=\left\{a_{1}, \ldots, a_{n}, \bar{a}_{1}, \ldots, \bar{a}_{n}\right\}$ of cardinality $\delta(*)=2 n$. If we write the path class of the edge $a_{i}$ still by $a_{i}$, then the fundamental group $\pi_{1}\left(R_{n}, *\right)=$ $\left\langle a_{1}, \ldots, a_{n}\right\rangle \cong F_{n}$, and $f_{*}: \pi_{1}\left(R_{n}, *\right) \rightarrow \pi_{1}\left(R_{n}, *\right)$ defined by $f_{*}\left(a_{i}\right)=a_{i}^{2}$. Note that $f_{*}$ is not an automorphism, with $\operatorname{Fix}\left(f_{*}\right)=\{1\}$ and $2 n$ equivalence classes of attracting fixed points that are induced by the oriented edge $a_{i}, \bar{a}_{i} \in \Delta(*)$,

$$
W_{a_{i}}=f_{*}^{\infty}\left(a_{i}\right)=a_{i} a_{i} a_{i} \cdots, \quad W_{\bar{a}_{i}}=f_{*}^{\infty}\left(a_{i}^{-1}\right)=a_{i}^{-1} a_{i}^{-1} a_{i}^{-1} \cdots .
$$

Therefore, $a(*)=a\left(f_{*}\right)=2 n, \operatorname{rkFix}\left(f_{*}\right)+a\left(f_{*}\right) / 2=n$ and

$$
\operatorname{ind}(*)=\operatorname{ichr}(*)=1-\operatorname{rkFix}\left(f_{*}\right)-a\left(f_{*}\right)=1-2 n .
$$

Next example shows that the inequality in Theorem 1.8 can be strict.

Example 6.2. Let $f:\left(R_{2}, *\right) \rightarrow\left(R_{2}, *\right)$ be a $\pi_{1}$-injective selfmap of the graph $R_{2}$ with one vertex $*$ and two edges $a_{1}, a_{2}$, such that $f\left(a_{1}\right)=a_{1}$ and $f\left(a_{2}\right)=\bar{a}_{2} a_{1} a_{2}$.

Note that $f$ is an RTT map with $Z=R_{2}$ and $Z_{0}=a_{1}$. Then $*$ is a nonempty fixed point class of $f$, and $\Delta(*)=\left\{\bar{a}_{2}\right\}$. If we write the path class of the edge $a_{i}$ still by $a_{i}$, then the fundamental group $\pi_{1}\left(R_{2}, *\right)=\left\langle a_{1}, a_{2}\right\rangle \cong F_{2}$, with $f_{*}: \pi_{1}\left(R_{2}, *\right) \rightarrow \pi_{1}\left(R_{2}, *\right)$ defined by $f_{*}\left(a_{1}\right)=a_{1}$ and $f_{*}\left(a_{2}\right)=a_{2}^{-1} a_{1} a_{2}$. Note that $f_{*}$ is not an automorphism, with $\operatorname{Fix}\left(f_{*}\right)=\left\langle a_{1}\right\rangle \cong \mathbb{Z}$ and the only equivalence class of attracting fixed points that is induced by the oriented edge $\bar{a}_{2} \in \Delta(*)$

$$
W_{\bar{a}_{2}}=f_{*}^{\infty}\left(a_{2}^{-1}\right)=a_{2}^{-1} a_{1}^{-1} a_{2} a_{1}^{-1} a_{2}^{-1} a_{1} a_{2} \cdots,
$$

Therefore, $\operatorname{rkFix}\left(f_{*}\right)+a\left(f_{*}\right) / 2=1+1 / 2<2$ and

$$
\operatorname{ind}(*)=\operatorname{ichr}(*)=1-\operatorname{rkFix}\left(f_{*}\right)-a\left(f_{*}\right)=-1 .
$$

Finally, we give two examples that support Conjecture 1.4 for empty and nonempty fixed point classes with indices 0 .

Example 6.3. Let $f:\left(R_{2}, *\right) \rightarrow\left(R_{2}, *\right)$ be a $\pi_{1}$-injective selfmap of the graph $R_{2}$ with one vertex $*$ and two edges $a, b$, such that $f(a)=b$ and $f(b)=\bar{a}$.

Below we will show that the fixed point class $\mathbf{F}_{a}$ associated to the $f$-route $a$ is empty with $\operatorname{ind}\left(\mathbf{F}_{a}\right)=\operatorname{ichr}\left(\mathbf{F}_{a}\right)$.

Indeed, fix a universal covering $q: \tilde{R}_{2} \rightarrow R_{2}$ with a given point $\tilde{*} \in q^{-1}(*)$, and a lifting $\tilde{a}:(I, 0,1) \rightarrow\left(\tilde{R}_{2}, \tilde{*}, \tilde{a}(1)\right)$ of the loop $a$. Then the lifting $\tilde{f}: \tilde{R}_{2} \rightarrow \tilde{R}_{2}$ defined by $\tilde{f}(\tilde{*})=\tilde{a}(1)$ is a composition of a rotation and a translation, so it is an isometry of the tree $\tilde{R}_{2}$ equipped with the natural metric $d$ such that each edge has length 1 . Note that $d(\tilde{f}(\tilde{*}), \tilde{*})=d(\tilde{a}(1), \tilde{*})=1$, so for any point $x \in \tilde{R}_{2}$,

$$
d(\tilde{f}(x), \tilde{*})=d(\tilde{f}(x), \tilde{f}(\tilde{*})) \pm d(\tilde{f}(\tilde{*}), \tilde{*})=d(x, \tilde{*}) \pm 1 .
$$

It implies that $\tilde{f}(x) \neq x$ and hence Fix $\tilde{f}=\emptyset$, namely, the fixed point class $\mathbf{F}_{a}$ is empty. 
On one hand, the $f$-route $a$ induces an injective endomorphism

$$
f_{a}: \pi_{1}\left(R_{2}, *\right) \rightarrow \pi_{1}\left(R_{2}, *\right), \quad a \mapsto a b a^{-1}, b \mapsto a^{-1},
$$

with $\operatorname{Fix}\left(f_{a}\right)=\left\langle a b a^{-1} b^{-1}\right\rangle \cong \mathbb{Z}$. On the other hand, consider $f_{*}: \pi_{1}\left(R_{2}, *\right) \rightarrow$

$\pi_{1}\left(R_{2}, *\right)$ defined by $a \mapsto b, b \mapsto a^{-1}$. Then for any infinite word $W \in \partial \pi_{1}\left(R_{2}, *\right)$ with initial segment $\left|W_{i}\right|=i$, the word length $\left|f_{*}\left(W_{i}\right)\right|=i$, and

$$
\left|f_{a}\left(W_{i}\right)\right|=\left|a f_{*}\left(W_{i}\right) a^{-1}\right| \leq i+2 .
$$

It follows that $f_{a}$ has no attracting fixed words. Therefore, $a\left(f_{a}\right)=0$.

By the arguments above, for the empty fixed point class $\mathbf{F}_{a}$ defined by the $f$-route $a$, we have

$$
\operatorname{ind}\left(\mathbf{F}_{a}\right)=\operatorname{ichr}\left(\mathbf{F}_{a}\right)=1-\operatorname{rkFix}\left(f_{a}\right)-a\left(f_{a}\right)=0 .
$$

Example 6.4 ([[33]). Let $f:\left(R_{2}, *\right) \rightarrow\left(R_{2}, *\right)$ be a $\pi_{1}$-injective selfmap of the graph $R_{2}$ with one vertex $*$ and two edges $a, b$, such that the induced endomorphism of $\pi_{1}\left(R_{2}, *\right)$ is given by $f_{*}(a)=a^{-1}$ and $f_{*}(b)=a^{-1} b^{2}$.

It is known from [J3] that $f$ has two nonempty fixed point classes and both of them have indices zero and hence both are inessential.

Note that $\operatorname{Fix}\left(f_{*}\right)=\{1\}$ and $f_{*}$ has an attracting fixed word

$$
f_{*}^{\infty}\left(b^{-1}\right)=b^{-2} a b^{-4} a b^{-2} a \cdots .
$$

Hence, $a\left(f_{*}\right)=1$ and the nonempty fixed point class consisting of $\{*\}$ has

$$
\operatorname{ind}(*)=\operatorname{ichr}(*)=1-\operatorname{rkFix}\left(f_{*}\right)-a\left(f_{*}\right)=0 .
$$

\section{REFERENCES}

[BH] M. Bestvina and M. Handel, Train tracks and automorphisms of free groups, Annals of Mathematics, 135 (1992), 1-51.

[C] D. Cooper, Automorphisms of free groups have finitely generated fixed point sets, J. Algebra 111 (1987), 453-456.

[CDP] M. Coornaert, T. Delzant and A. Papadopoulos, Géométrie et théorie des groupes, Lecture Notes in Math. 1441, Springer-Verlag, Berlin, 1990.

[DV] W. Dicks and E. Ventura, The group fixed by a family of injective endomorphisms of a free group, Contemporary Mathematics vol. 195, American Mathematical Society, Providence (1996).

[GJLL] D. Gaboriau, A. Jaeger, G. Levitt and M. Lustig, An index for counting fixed points for automorphisms of free groups, Duke Math. J. 93 (1998), no. 3, 425-452.

[J1] B. Jiang, Lectures on Nielsen Fixed Point Theory, Contemporary Mathematics vol. 14, American Mathematical Society, Providence (1983).

[J2] B. Jiang, Bounds for fixed points on surfaces, Math. Ann., 311 (1998), 467-479.

[J3] B. Jiang, Fixed points and braids, Invent. Math. 75 (1984) 69-74.

[JWZ] B. Jiang, S. Wang and Q. Zhang, Bounds for fixed points and fixed subgroups on surfaces and graphs, Alg. Geom. Topology, 11 (2011), 2297-2318.

[Sh1] H. Short, Quasiconvexity and a theorem of Howson's, Group Theory from a Geometrical Viewpoint (Trieste, 1990), World Sci., River Edge, N.J., 1991, 168-176.

[Sh2] H. Short et al., Notes on word hyperbolic groups, Group Theory from a Geometrical Viewpoint (Trieste, 1990), World Sci., River Edge, N.J., 1991, 3-63. 
[Z] Q. Zhang, Bounds for fixed points on Seifert manifolds, Topology Appl. 159 (15) (2012), 3263-3273.

School of Mathematics and Statistics, Xi' an Jiaotong University, Xi' an 710049, China

E-mail address: zhangq.math@mail.xjtu.edu.cn

Department of Mathematics, Capital Normal University, Beijing 100048, China

E-mail address: zhaoxve@mail. cnu.edu.cn 